ARTICLE

Received 22 Aug 2016 | Accepted 7 Apr 2017 | Published 6 Jun 2017 DOl: 10.1038/ncomms15558 OPEN

\title{
Loss of function $\mathrm{CHCHD10}$ mutations in cytoplasmic TDP-43 accumulation and synaptic integrity
}

Jung-A. A. Woo ${ }^{1,2, \star}$, Tian Liu ${ }^{1,2, \star}$, Courtney Trotter ${ }^{1,2, \star}$, Cenxiao C. Fang ${ }^{1,2}$, Emillio De Narvaez ${ }^{1,2}$, Patrick LePochat ${ }^{1,2}$, Drew Maslar ${ }^{1,2}$, Anusha Bukhari ${ }^{1,2}$, Xingyu Zhao ${ }^{1,2}$, Andrew Deonarine ${ }^{3}$, Sandy D. Westerheide ${ }^{3} \&$ David E. Kang ${ }^{1,2,4}$

Although multiple CHCHD10 mutations are associated with the spectrum of familial and sporadic frontotemporal dementia-amyotrophic lateral sclerosis (FTD-ALS) diseases, neither the normal function of endogenous $\mathrm{CHCHD10}$ nor its role in the pathological milieu (that is, TDP-43 pathology) of FTD/ALS have been investigated. In this study, we made a series of observations utilizing Caenorhabditis elegans models, mammalian cell lines, primary neurons and mouse brains, demonstrating that $\mathrm{CHCHD10}$ normally exerts a protective role in mitochondrial and synaptic integrity as well as in the retention of nuclear TDP-43, whereas FTD/ALS-associated mutations (R15L and S59L) exhibit loss of function phenotypes in C. elegans genetic complementation assays and dominant negative activities in mammalian systems, resulting in mitochondrial/synaptic damage and cytoplasmic TDP-43 accumulation. As such, our results provide a pathological link between $\mathrm{CHCHD10}$-associated mitochondrial/synaptic dysfunction and cytoplasmic TDP-43 inclusions.

\footnotetext{
${ }^{1}$ USF Health Byrd Alzheimer's Institute, University of South Florida, Morsani College of Medicine, Tampa, Florida 33613, USA. ${ }^{2}$ Department of Molecular Medicine, University of South Florida, Morsani College of Medicine, Tampa, Florida 33613, USA. ${ }^{3}$ Department of Cell Biology, Microbiology \& Molecular Biology, University of South Florida, College of Arts and Sciences, Tampa, Florida 33620, USA. ${ }^{4}$ James A. Haley Veteran's Administration Hospital, Tampa, Florida 33612, USA. * These authors contributed equally to this work. Correspondence and requests for materials should be addressed to D.E.K. (email: dkang@health.usf.edu).
} 
F rontotemporal dementia (FTD) is a progressive neurodegenerative disease classically characterized by the selective degeneration of the frontal and temporal lobes, associated with executive impairments, changes in personality and language dysfunction. It is now clear, however, that FTD is frequently accompanied by motor neuron disease or amyotrophic lateral sclerosis (ALS), and common genetic mutations in multiple genes have been associated with both FTD and ALS ${ }^{1,2}$. Indeed, $\sim 15 \%$ of FTD patients also suffer from ALS, and up to $50 \%$ of classical ALS patients also suffer from symptoms of FTD or mild cognitive impairment ${ }^{2}$. The unifying pathological link between the vast majority of ALS and FTD patients is the accumulation of cytoplasmic TDP-43 inclusions, the latter in the majority FTD subtype, frontotemporal lobar degeneration with TDP-43 pathology (FTLD-TDP) ${ }^{3,4}$.

TAR DNA-binding protein 43 (TDP-43) is a nuclear protein in the family of heterogeneous ribonucleoproteins (hnRNPs) that plays a major role in regulating RNA splicing, stability and transport ${ }^{5}$. However, in pathological neurons, TDP-43 is often found in the cytoplasm in a ubiquitinated and fragmented form, which are prone to aggregation ${ }^{6-8}$. While TDP- 43 mutations are found in a small proportion of ALS and FTLD-TDP, TDP-43 pathology is associated with the vast majority of ALS and $\mathrm{FTLD}^{4,9}$. Increasing evidence indicates that TDP-43 is highly neurotoxic, in large part, by inducing mitochondrial dysfunction ${ }^{10-12}$. Specifically, TDP-43 is co-localized with mitochondria and reduces mitochondrial length by promoting mitochondrial fission, resulting in mitochondrial transport defects $^{10}$. The mitochondrial fusion protein Mfn2 prevents mitochondrial fragmentation, superoxide production and depolarization $(\Delta \Psi \mathrm{m})$ induced by TDP-43 mutations $^{10}$, and blockade of TDP-43 translocation to mitochondria also blocks its neurotoxicity ${ }^{12}$. In line with these observations, TDP-43 overexpression in transgenic mice increases mitochondrial fission proteins Drp1 and Fis1, reduces the mitochondrial fusion protein Mfn1, and promotes mitochondrial fragmentation and aggregation $^{11}$.

Coiled-coil-helix-coiled-coil-helix domain containing 10 (CHCHD10) encodes a protein that is localized to the intermembrane space of mitochondria ${ }^{13}$ associated with the mitochondrial contact site and cristae organizing system (MICOS) together with mitofusin, mitophilin, CHDHD3 and

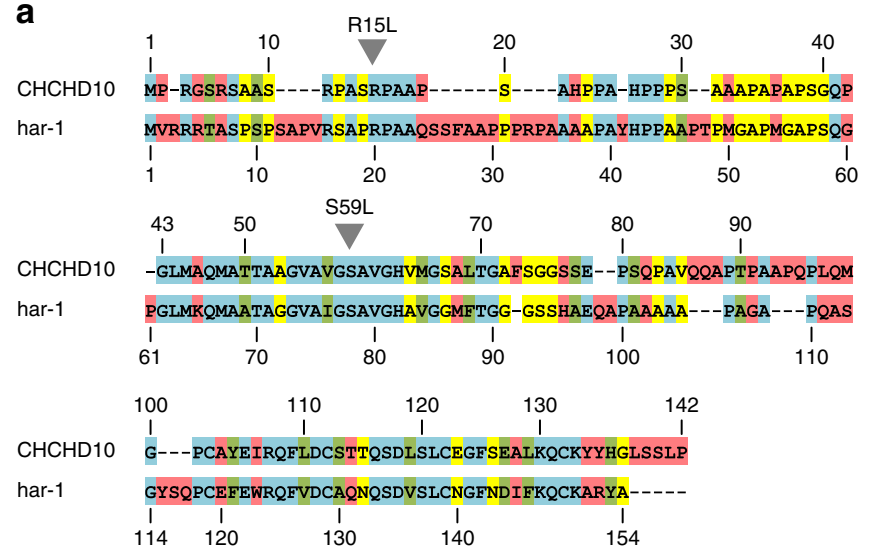

Identical
Conserved Semi-conserved

\section{Not}

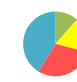

b

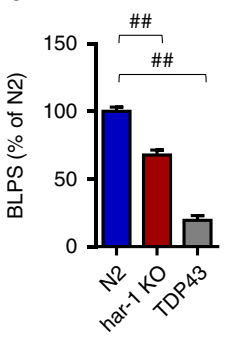

C
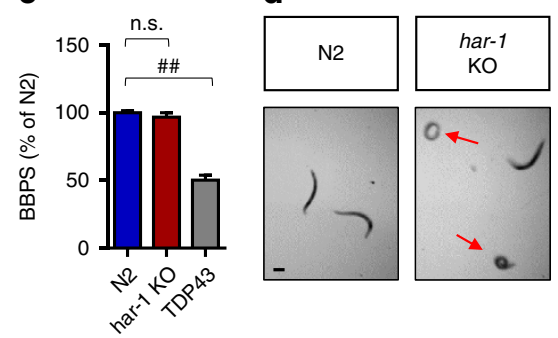

e

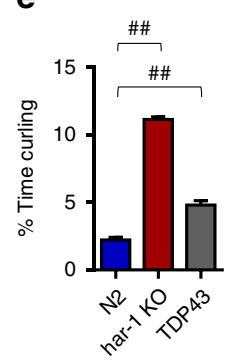

f

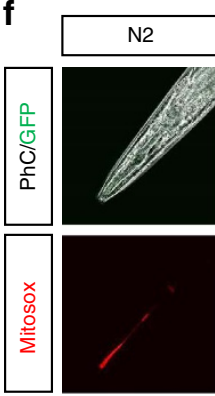

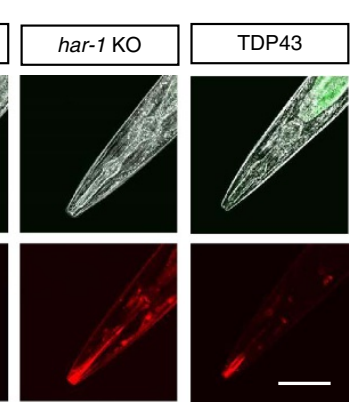
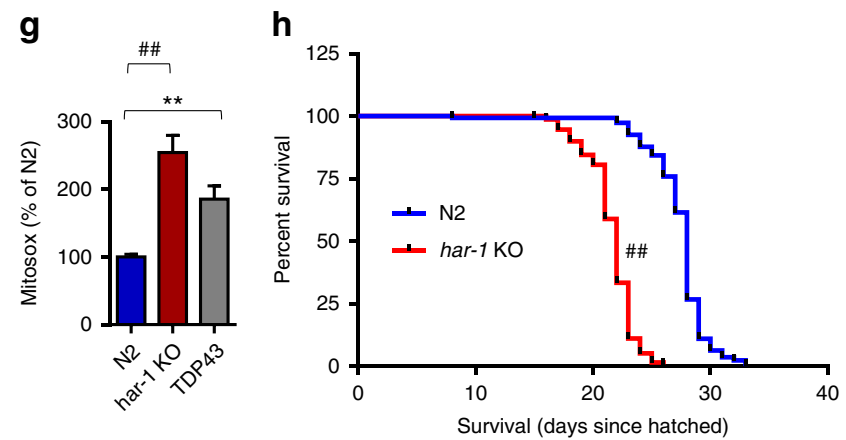

Figure 1 | Loss of har-1 impairs locomotion mitochondrial health and longevity in C. elegans. (a) Protein sequence alignment between human CHCHD10 and C. elegans har- 1 . Note that C. elegans har- 1 shares $41 \%$ identity, $12 \%$ conserved similarity, and $18 \%$ semi-conserved similarity to human $\mathrm{CHCHD} 10$ (http://useast.ensembl.org/index.html). (b-e) Age-synchronized worms per strain (3-day adults: N2, har-1 KO (har-1 ${ }^{-/}{ }^{-}$) and TDP43) transferred to fresh NGM plate or M9 buffer and videotaped to measure motility, liquid thrashing rate, and curling behaviour (1-way ANOVA, post hoc Tukey,

$\left.{ }^{\# \#} P<0.0001\right)$. (b) Motility (measured in BLPS) on NGM plate measured at ambient room temperature $\left(22^{\circ} \mathrm{C}\right.$ ) and normalized to N2 control from 90 to 94 worms per strain. (c) Liquid thrashing rate (BBPS) measured in M9 buffer at ambient room temperature $\left(22^{\circ} \mathrm{C}\right.$ ) and normalized to $\mathrm{N} 2 \mathrm{control}$ from 50 worms per strain. (d,e) Representative images of curling posture (red arrows). Per cent time spent curling measured at ambient room temperature ( $22^{\circ} \mathrm{C}$ ) from 50 worms per strain (scale bar, $100 \mu \mathrm{m})$. (f,g) Age-synchronized worms stained with $5 \mu \mathrm{M}$ mitosox-red in M9 buffer for 45 min, mounted live on agar pads, imaged by confocal microscopy, quantified with Image J, and normalized to N2 control (one-way ANOVA, ${ }^{\star \star} P<0.01,{ }^{\# \#} P<0.0001, n=29$ worms/ strain, scale bar, $100 \mu \mathrm{m}$ ). Average intensity of head region excluding mouth, pharynx, and intestine quantified. (h) Lifespan analysis (\% of worms surviving since days hatching) of $\mathrm{N} 2$ and har- $1 \mathrm{KO}$ worms at $16^{\circ} \mathrm{C}$ (Log-rank Mantel-Cox test, $\# \#<0.0001, n=170$ worms/strain). All quantitative graphs with error bars were expressed as mean \pm s.e.m. ANOVA, analysis of variance; $\mathrm{KO}$, knockout. 
CHCHD6 (ref. 14). Recent human genetic studies have identified multiple $\mathrm{CHCHD} 10$ mutations in sporadic and familial FTD-ALS spectrum disorders from independent cohorts and ethnic groups ${ }^{13-17}$, implicating the critical role of mitochondria in FTD-ALS diseases. CHCHD10 mutations have also been associated with Charcot-Marie-Tooth disease type 2 (ref. 18), mitochondrial myopathy and spinal muscular atrophy Jokela type $^{19}$. However, despite the role of the FTD/ALS-linked CHCHD10 S59L mutation in reducing mitochondrial length and disorganization of mitochondrial cristae morphology ${ }^{13}$, neither the normal function of endogenous CHCHD10 nor the nature of the FTD/ALS CHCHD10 mutations has been investigated. Moreover, whether and how CHCHD10 mutations alter the pathological landscape (that is, TDP-43 inclusions) of FTD-ALS spectrum diseases is completely unknown, and no FTD/ALS mutations other than S59L have been functionally characterized. Here we show in Caenorhabditis elegans models, cultured cells, primary neurons, and mouse brains that CHDHD10 normally plays a neuroprotective role and that FTD/ALS CHCHD10 mutations (R15L and S59L) display loss of function phenotypes and function in concert with TDP-43 to induce its cytoplasmic mislocalization, resulting in mitochondrial and synaptic damage.

\section{Results}

Loss of har-1 loss impairs movement and mitochondria in C. elegans. The C. elegans har-1 gene is the closest orthologue to mammalian $\mathrm{CHCHD} 10$ with $41 \%$ identity, $12 \%$ conserved homology and $18 \%$ semi-conserved homology to human $\mathrm{CHCHD} 10$ at the level of amino acid sequence (Fig. 1a). The R15 and $\mathrm{S} 59 \mathrm{CHCHD} 10$ residues mutated in FTD/ALS are conserved between $C$. elegans har-1 and human CHCHD10 proteins (Fig. 1a). To gain insights to the function of CHCHD10 in vivo, we utilized the VC3169 har-1 ${ }^{-1-}$ C. elegans model $(g k 3124)$ to assess locomotion in comparison with wild-type N2 ancestral and TDP-43 transgenic strains. TDP-43 expression in the TDP-43 transgenic C. elegans CL6049 is driven by the neuron-specific $s n b-1$ promoter, a strain with impairments in locomotion at room temperature that worsens with heat exposure ${ }^{20}$. In motility tests at ambient temperature, we observed significantly slower rates of movement in both har-1 $-1-$ and TDP-43 transgenic worms by 32 and $80 \%$, respectively, as compared to N2 (Fig. 1b). In liquid thrashing tests at ambient temperature, har-1 ${ }^{-1}$ - worms showed no significant reduction in body bends per second (BBPS), whereas the TDP-43 worms displayed a significant $50 \%$ reduction in BBPS (Fig. 1c). Upon exposure to heat $\left(30^{\circ} \mathrm{C}\right.$ for $\left.30 \mathrm{~min}\right)$, both TDP-43 and har-1 ${ }^{-1}$ strains showed dramatically reduced liquid thrashing rates compared to N2 controls (Supplementary Fig. 1a), indicating that heat exposure renders the har-1-1strain more vulnerable to the loss of har-1 possibly due to proteostatic stress. Next, we tested the strains for the percentage of time spent in a curled posture in liquid curling tests, since both TDP-43 and har-1 $1^{-/-}$worms showed salient abnormalities in their pattern of thrashing in liquid (Fig. 1d, Supplementary Fig. $1 \mathrm{~b}$ and Supplementary Movies 1,2). Interestingly, abnormal curling behaviour increases with age in C. elegans and is reflective of a defect in motor coordination ${ }^{21}$. At ambient temperature, har- $1^{-1}-$ and TDP-43 strains showed 4.9-fold and 2.1 increases in time spent in curled posture while thrashing in liquid, respectively (Fig. 1e). This differential phenotype was especially accentuated in the TDP-43 worms when exposed to $30^{\circ} \mathrm{C}$ (Supplementary Fig. 1c). As har-1 is a mitochondrial protein ${ }^{22}$, we tested the hypothesis that mitochondrial dysfunction might

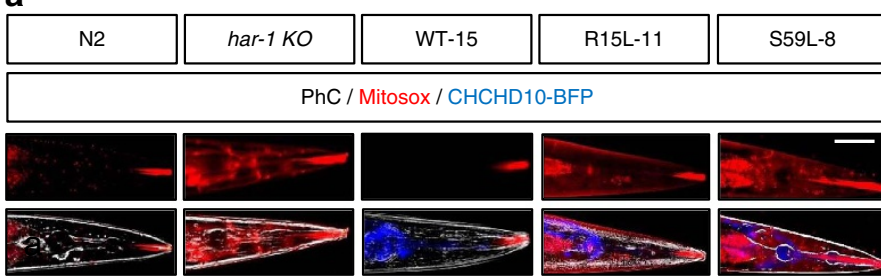

b

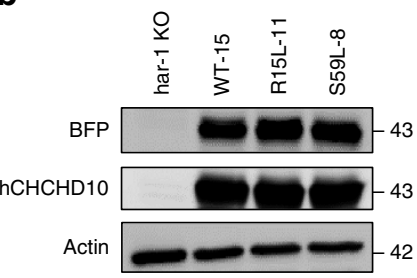

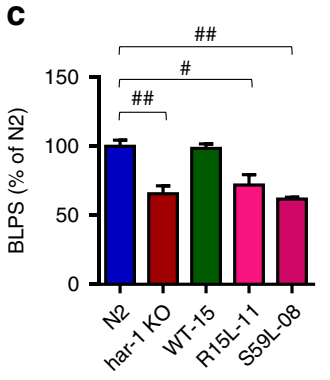

d

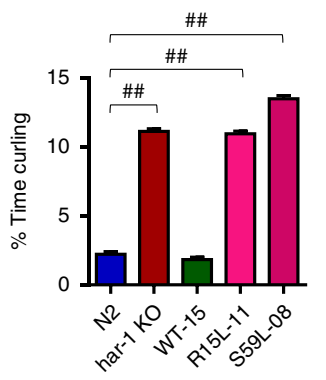

e

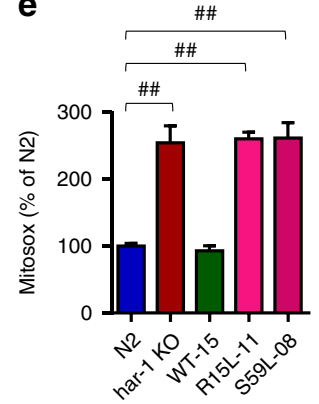

f

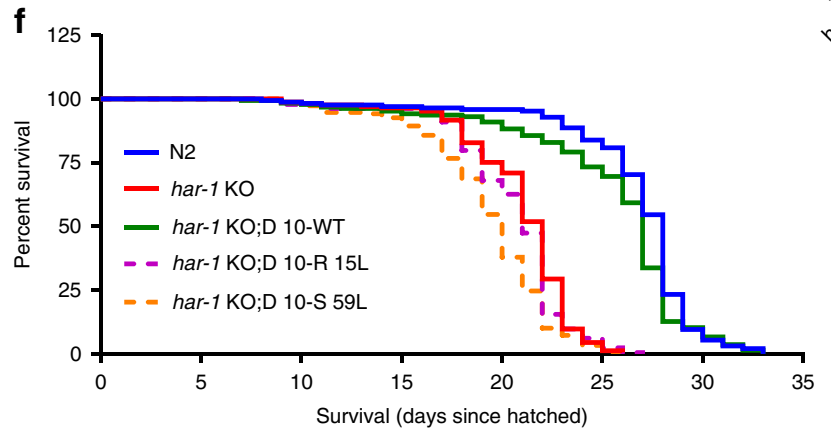

Figure 2 | Wild-type human CHCHD10 but not FTD/ALS mutations complement the loss of har-1. (a) Representative images of age-synchronized worms stained with $5 \mu \mathrm{M}$ mitosox-red and imaged for BFP (CHCHD10), mitosox-red and phase contrast (PhC) by confocal microscopy. Scale bar, $100 \mu \mathrm{m}$. (b) Representative blots of BFP and hCHCHD10 expression by immunoblotting. (c,d) Age-synchronized N2, har-1 KO and har-1 KO worms expressing WT, R15L or S59L CHCHD10 transferred to fresh NGM plate or M9 buffer and videotaped to measure motility (BLPS, $n=50$ worms/strain) and curling behaviour ( $n=50$ worms/strain) at normalized to N2 control (one-way ANOVA, post hoc Tukey, ${ }^{\#} P<0.001,{ }^{\#} p<0.0001$ ). (e) Mitosox-red intensity (head region excluding mouth, pharynx and intestine) at $22^{\circ} \mathrm{C}$ normalized to $\mathrm{N} 2$ control in adult day 3 worms (one-way ANOVA, post hoc Tukey, ${ }^{\# \#} P<0.0001, n=29$ worms/strain). (f) Lifespan analysis (\% of worms surviving since days hatched) of N2, har-1 KO, har-1 KO worms expressing $\mathrm{CHCHD} 10$ variants at $16^{\circ} \mathrm{C}$ (data from WT-2/WT-14/WT-15 combined, R15L-3/R15L-10/R15L-11 combined and S59L-2/S59L-18/S59L-08 combined) (Log-rank Mantel-Cox test, ${ }^{\# \#} P<0.0001, n=170-190$ worms/genotype). All quantitative graphs with error bars were expressed as mean \pm s.e.m. ANOVA, analysis of variance; KO, knockout. 
underlie the loss of har-1. We therefore subjected the strains to mitosox-red staining, which indicates the level of mitochondrial superoxide, with higher levels of superoxide corresponding to poorer mitochondrial health. Quantification of mitosox-red intensity in the head region (excluding mouth, pharynx and intestines; Supplementary Fig. 1d) on adult day 3 demonstrated significantly increased mitochondrial superoxide in both TDP-43 and $h a r-1^{-1-}$ strains (Fig. 1f,g). Despite the increase in mitosoxred intensity in har-1 ${ }^{-}$- worms on adult days 3 and 7 , no significant differences in mitosox-red were seen on adult day 1 compared to N2 controls (Supplementary Fig. 1e), indicative of an aging but not developmental alteration. Given the important role of mitochondrial health in $C$. elegans longevity ${ }^{23}$, we performed lifespan analysis of age-matched $\mathrm{N} 2$ and har1 ${ }^{-1}-$ strains at $16^{\circ} \mathrm{C}$. While all $\mathrm{N} 2$ worms died over a 33-day period after hatching, the har-1-1- strain showed a significantly reduced lifespan (median survival 22 days) compared to N2 controls (median survival 28 days; Fig. 1h), indicating that endogenous har-1 contributes to lifespan of C. elegans.

Human CHCHD10 but not FTD/ALS mutations complement the loss of har-1. To determine whether human CHCHD10 can complement phenotypes in the $h a r-1^{-1}$ background, we engineered $C$. elegans DNA constructs expressing human $\mathrm{CHCHD10-blue} \mathrm{fluorescent} \mathrm{protein} \mathrm{(BFP)} \mathrm{variants} \mathrm{under} \mathrm{the}$ control of the ubiquitous eef-1A.1 promoter. These constructs were then microinjected into the gonad of har-1 $-1-C$. elegans. Upon verification of generational transmission by BFP fluorescence in their progeny (Fig. 2a), locomotion, mitosox-red, and longevity assays were carried out. We initially chose three independent har-1 (gk3124) rescue lines (WT-02, WT-14 and WT-19) with moderate to high BFP fluorescence. In all three clonal strains, crawling speeds were significantly rescued by human CHCHD10 (Supplementary Fig. 2a). In liquid curling tests, abnormal curling was also rescued in all three hCHCHD10expressing strains (Supplementary Fig. 2b). Consistent with these data, the har-1-l-(CHCHD10-02) strain, which exhibited moderate BFP fluorescence, also fully reduced mitosox-red staining to N2 control levels (Supplementary Fig. 2c). Indeed, we confirmed the expression of CHCHD10 by western blotting using antibodies against BFP and hCHCHD10 (Supplementary Fig. 2d).

We next injected har-1 $1^{-/-}$strains with WT and FTD/ALSlinked CHCHD10 mutations (R15L and S59L) and chose strains that contained comparable BFP fluorescence and CHCHD10/BFP expression by immunoblotting (Fig. 2a,b). WT but not R15L or S59L strains rescued the slow motility and abnormal curling phenotypes associated with har-1 ${ }^{-1}-($ Fig. $2 \mathrm{c}, \mathrm{d})$, indicating that both mutations exhibit a loss of function phenotype with regards to har-1 complementation. While WT hCHCHD10 reduced mitochondrial superoxide (mitosox-red) down to control N2 levels, R15L and S59L mutants completely failed to rescue this phenotype (Fig. 2a,e). Similar results on crawling, curling, and mitosox-red tests were obtained from additional clonal strains (Supplementary Fig. 2d-i). Interestingly, the S59L-18 mutant showed a curling phenotype that was significantly worse than the parental har-1 ${ }^{-1}-$ strain (Supplementary Fig. 2g), suggesting that the S59L mutation may also contains an additional gain-offunction component. Likewise, in liquid thrashing tests, both R15L and S59L mutants showed a modest but significant decline in thrashing rates compared the parental har- $1^{-1-}$ strain (Supplementary Fig. 2h). Lifespan analysis at $16^{\circ} \mathrm{C}$ showed that while three strains of WT hCHCHD10 (WT-02, WT-14 and WT15 combined) prolonged lifespan to control N2 levels, 3 strains of R15L (R15L-03, R15L-10 and R15L-11 combined) and S59L (S59L-02, S59L-18 and S59L-08 combined) failed to rescue the reduced lifespan phenotype in the har-1-/- background (Fig. 2f). The median survival times for $\mathrm{N} 2$ and $h a r-1^{-1-}$; D10-WT strains were 27-28 days, whereas those for har-1 ${ }^{-1-}$ (22 days), har-1 $1^{-1-}$;D10-R15L (21 days), and har-1 ${ }^{-1-}$; D10S59L (20 days) were significantly shorter.

CHCHD10 dysfunction promotes mitochondrial and synaptic deficits. Bannwarth et al. ${ }^{13}$ reported that the CHCHD10 S59L mutation reduces mitochondrial length and promotes disorganization of mitochondrial cristae structure. However, the effects of endogenous CHCHD10 depletion have not been investigated in mammalian cells. Thus, we utilized lentiviruses expressing mouse CHCHD10 shRNA together with green fluorescent protein (GFP) or GFP alone to knockdown endogenous CHCHD10 in mouse HT22 cells, a hippocampusderived neuroblastoma cell line that has been characterized for mitochondrial dysfunction and cell death ${ }^{24,25}$. Forty-eight hours after transduction, immunoblotting indeed confirmed that CHCHD10 shRNA lentivirus transduced cells contained markedly less endogenous $\mathrm{CHCHD} 10$ by $>60 \%$ overall (Fig. 3a,b). Next, we measured mitochondrial superoxide (mitosox-red) and membrane potential ( $\Delta \Psi \mathrm{m}, \mathrm{JC}-1$ aggregates) in GFP expressing HT22 cells. JC-1 aggregates indicate healthy polarized mitochondria (red), whereas JC-1 monomers (green) indicate unhealthy depolarized mitochondria ${ }^{26}$. Although we were unable to measure JC-1 monomers due to its spectral overlap with GFP fluorescence, depletion of endogenous CHCHD10 by shRNA in GFP + cells significantly increased mitosox-red intensity by $>2$-fold (Fig. 3c,d) and reduced JC-1 aggregates by $\sim 50 \%$ (Fig. $3 \mathrm{c}-\mathrm{e}$ ). This phenotype was not attributable to the related gene $\mathrm{CHCHD} 2$, as knockdown of CHCHD10 did not change CHCHD2 mRNA as measured by quantitative PCR reverse-transcription (qRT-PCR) (Supplementary Fig. 3a,b). Real-time qRT-PCR analysis of 15 genes encoded in mitochondria also demonstrated that knockdown of endogenous CHCHD10 (Fig. 3f,g) reduced levels of all 15 transcripts by $40-75 \%$ and significantly reduced mitochondrial transcripts overall (Fig. 3h), indicating a general perturbation in mitochondrial health.

To visualize the localization of $\mathrm{CHCHD} 10$ and assess mitochondrial integrity secondary to WT or FTD/ALS mutant CHCHD10 expression, we next co-transfected NIH3T3 cells with the mitochondrial marker mito-dsRed and wild-type (WT) or FTD/ALS-linked (R15L or S59L) CHCHD10 variants. We chose the NIH3T3 cells for this purpose due to their larger size and flatter shape than HT22 cells to better visualize mitochondrial morphology. As anticipated, all CHCHD10 variants were largely co-localized with mito-dsRed (Fig. 3i). Whereas WT CHCHD10 showed visibly elongated mitochondria, R15L and S59L CHCHD10 mutants displayed significantly fragmented and shortened mitochondria (Fig. 3i,j), which often did not colocalize with mito-dsRed. Quantification showed that the R15L and S59L mutants co-localized significantly less with mito-dsRed compared to its WT counterpart (Fig. 3i-1). Upon treatment with the mitochondrial uncoupling agent FCCP $(1 \mu \mathrm{M})$ for $1 \mathrm{~h}$, fragmentation of mitochondria were seen in all conditions, although mitochondrial lengths were still shorter in R15L and S59L mutants compared to vector or WT CHCHD controls (Fig. 3i,k). WT CHCHD10 transfected cells showed a nonsignificant trend toward increased mitochondrial length compared to vector control transfected cells both at basal and carbonyl cyanide 4-(trifluoromethoxy)phenylhydrazone (FCCP) conditions (Fig. 3i-k). Real-time qRT-PCR analysis demonstrated that like CHCHD10 knockdown, R15L and S59L mutations significantly reduced mitochondrial transcripts overall compared to 
a

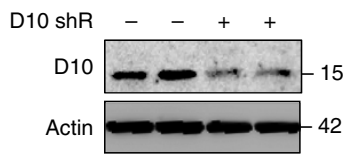

b

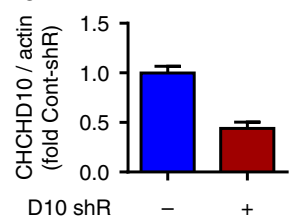

f

D10siR
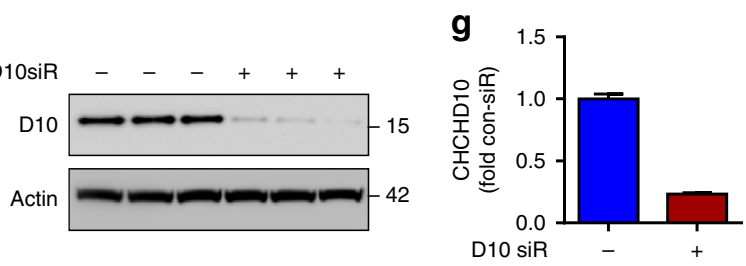

C

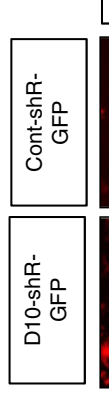

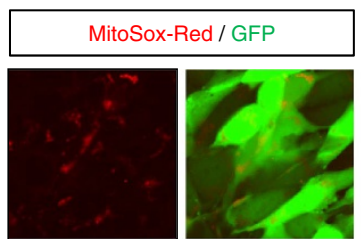

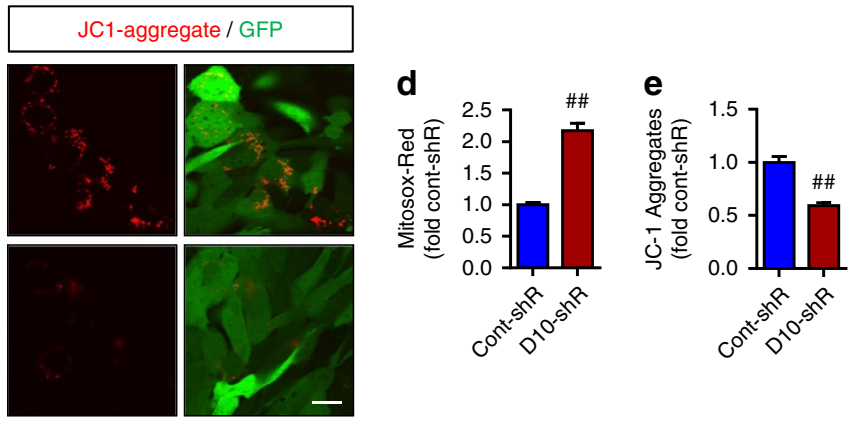

h
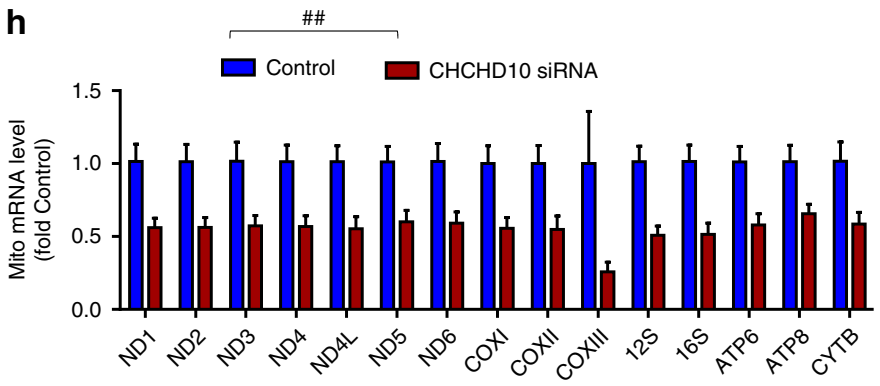

i

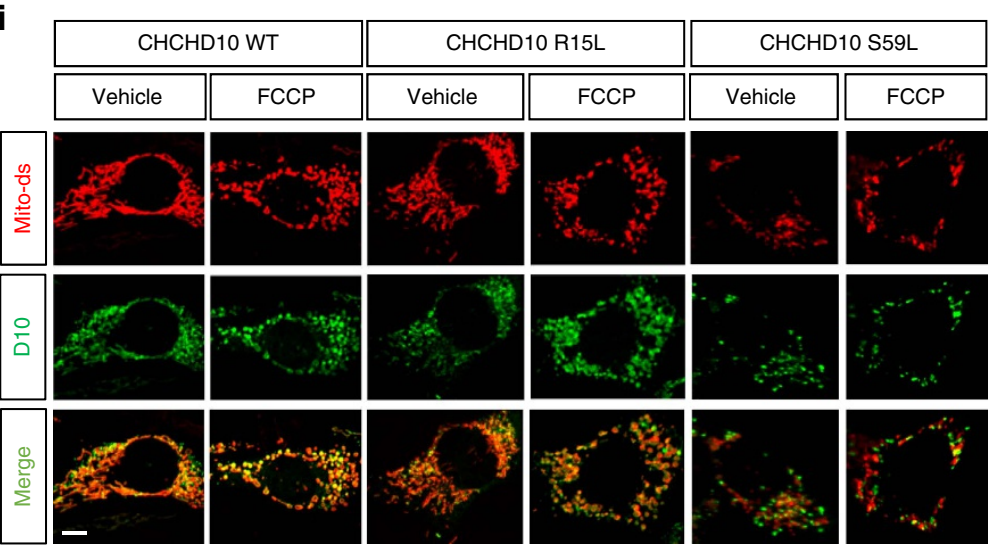

m
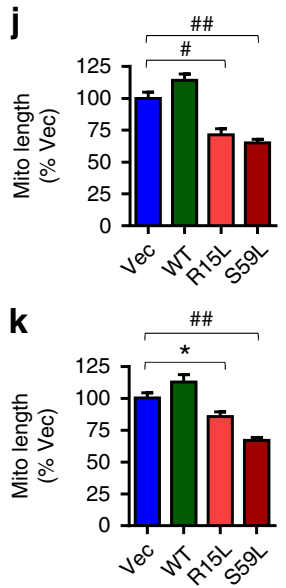

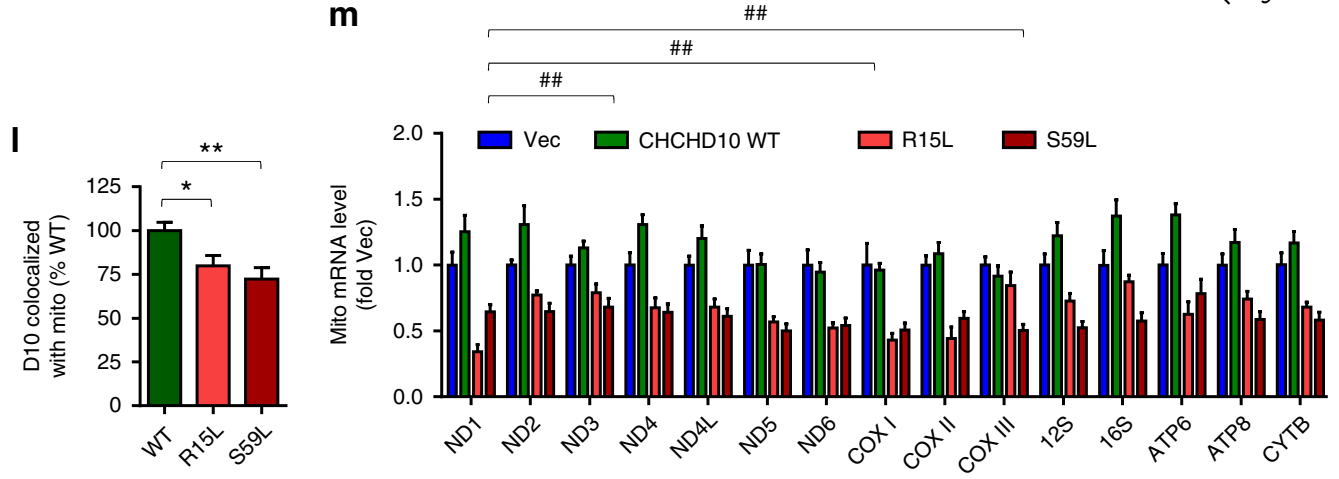

vector or WT controls (Fig. $3 \mathrm{~m}$ ). Fourteen out of 15 individual mitochondrial encoded transcripts were significantly reduced by the R15L mutation, and all 15 transcripts were significantly reduced by the S59L mutation compared to WT CHCHD10 (Fig. $3 \mathrm{~m}$ ). In contrast, WT CHCHD10 significantly increased mitochondrial transcripts overall compared to vector control (Fig. $3 \mathrm{~m}$ ) and showed a nonsignificant trend for increasing 11/15 transcripts individually compared to vector control (Fig. $3 \mathrm{~m}$ ), indicating that expression of WT CHCHD10 can augment endogenous CHCHD10 function, whereas R15L and
S59L mutations display a phenotype like that of CHCHD10 depletion.

Neuronal synapses are energy-intensive compartments highly enriched in mitochondria, which are transported in an activitydependent manner ${ }^{27}$. To determine whether the mitochondrial dysfunction associated with CHCHD10 can been seen at the level of synaptic integrity, we transduced DIV15 hippocampal primary neurons with control GFP or CHCHD10-shRNA/GFP lentiviruses. Compared to GFP only transduced neurons, CHCHD10-shRNA/GFP-transduced neurons showed significantly 
reduced intensity of drebrin (postsynaptic marker) and synaptophysin (presynaptic marker) puncta in neuronal processes on DIV21 (Fig. 4a,b). We also transduced primary neurons with rAAV9 expressing empty vector or CHCHD10 variants (WT, R15L and S59L). R15L or S59L transduced neurons showed significantly reduced drebrin and synaptophysin puncta compared to empty vector or WT CHCHD10 transduced neurons on DIV21 (Fig. 4c,d), indicating that these FTD/ALSlinked CHCHD10 mutants display a synaptic phenotype similar to that seen with CHCHD10 depletion.

TDP-43 interacts with CHCHD10 and promotes its nuclear localization. TDP-43 is normally a nuclear protein that can translocate to the cytoplasm and mitochondria under pathological conditions ${ }^{3,4}$. Thus, we first assessed whether endogenous CHCHD10 can form a physical complex with endogenous TDP-43. Whereas IgG beads alone failed to pull down either endogenous CHCHD10 or TDP-43, CHCHD10 immune complexes contained endogenous TDP-43, and TDP-43 immune complexes also contained endogenous CHCHD10 (Supplementary Fig. 4a). In HT22 cells transfected with vector, Flag-CHCHD10 and/or TDP-43-tomato, M2-Flag immune complexes contained TDP-43-tomato, whereas no TDP-43tomato was present in M2-Flag immune complexes from TDP-43-tomato only transfected cells (Fig. 5a). We next generated $\mathrm{CHCHD} 10$ constructs lacking N-terminal 16 residues $(\Delta 1-15)$ or lacking $C$-terminal residues $119-142(\Delta 119-142)$. Residues 1-15 contain a hypothetical mitochondrial targeting sequence ${ }^{28}$, whereas residues $119-142$ contain the $\mathrm{CX}_{9} \mathrm{CCX}_{9} \mathrm{C}$ structural motif (Fig. 5b). Co-transfection of CHCHD10 variants with vector control or TDP-43-tomato demonstrated that while WT and $\Delta 119-142$ variants similarly formed complexes with TDP-43, the $\Delta 1-15$ variant failed to interact with TDP-43 (Fig. 5c). Like WT CHCHD10, mutant CHCHD10 R15 and S59L formed complexes with TDP-43 (Supplementary Fig. 4b). Given the physical association of TDP-43 with CHCHD10 and the nuclear localization of TDP-43, we tested whether TDP-43 overexpression alters endogenous CHCHD10 localization. Surprisingly, TDP-43-tomato transfection increased nuclear localization of $\mathrm{CHCHD} 10$ by a significant three-fold while having a marginal effect on cytoplasmic CHCHD10 in biochemical fractionation experiments (Fig. 5d,e). The mitochondrial protein TOM20 and nuclear protein LaminB1 were not detected in nuclear and cytoplasmic fractions, respectively (Fig. 5d). In accord with biochemical observations, the ratio of endogenous nuclear versus cytoplasmic $\mathrm{CHCHD} 10$ was significantly increased by $\sim 2.3$-fold in TDP-43-tomato transfected cells as measured by immunofluorescence (Fig. 5f,g), while having no effect on endogenous CHCHD10 transcript (Fig. 5g, bottom graph).

CHCHD2, a protein with high homology to CHCHD10 and recently shown to be mutated in Parkinson's and Lewy body diseases $^{29-31}$, can shuttle between mitochondria and nucleus and function as a nuclear co-transactivator under conditions of hypoxia $^{32,33}$. Thus, we tested whether the sets of nuclear genes altered by $\mathrm{CHCHD} 2$ are also similarly altered by $\mathrm{CHCHD} 10$ knockdown or overexpression. Indeed, cytochrome c oxidase subunit-4 isoform-2 (COX4.2), NADH dehydrogenase ironsulfur protein 3 (NDUFS3), and NADH dehydrogenase- $1 \beta$ subcomplex subunit 6 (NDUFB6) transcripts (nuclear-encoded mitochondrial proteins) were all significantly reduced by CHCHD10 shRNA transduction (Fig. 5h), similar to that seen with $\mathrm{CHCHD} 2$ knockdown ${ }^{33}$. However, this was not attributable to $\mathrm{CHCHD} 2$ expression, as CHCHD10 knockdown had no effect on $\mathrm{CHCHD} 2$ transcript levels (Supplementary Fig.3b). Conversely, CHCHD10 overexpression significantly increased NDUFS3 and NDUFB6 transcripts, but such increases did not significantly differ among WT, R15 and S59L variants (Fig. 5i), suggesting that the putative nuclear transactivation function of CHCHD10 may not be profoundly altered by the presence of R15L and S59L mutations. Nonetheless, these novel data indicate that TDP-43 can form physical complexes with CHCHD10, alter its subcellular localization, and potentially regulate $\mathrm{CHCHD} 10$ mediated nuclear function.

CHCHD10 dysfunction induces cytoplasmic TDP-43 accumulation. To gain insights into the normal activity of CHCHD10 on TDP-43 localization, we transduced primary mouse hippocampal neurons with control GFP or CHCHD10 shRNA/GFP lentiviruses and subjected neurons for immunocytochemistry (ICC) for TOM20 (mitochondria marker-pseudo-coloured to green) and TDP-43 (red). In GFP only transduced neurons, endogenous TDP-43 was exclusively localized to the nucleus (Fig. 6a, left upper panel). However, in neurons transduced with CHCHD10 shRNA/GFP, endogenous TDP-43 was not only localized to the nucleus but also in the cytoplasm and neuritic processes (Fig. 6a, right upper panel). Quantification of nuclear and cytoplasmic TDP-43 staining demonstrated a significant $>30 \%$ increase in the ratio of cytoplasmic/nuclear TDP-43 in CHCHD10 shRNA/GFP compared to GFP-only transduced neurons (Fig. 6b). We also transduced primary neurons with rAAV9 expressing empty vector control, WT, R15L or S59L CHCHD10 variants. Compared to control and WT CHCHD10, neurons transduced with R15L or S59L CHCHD10 mutants also demonstrated significant increases in the ratios of endogenous cytoplasmic/nuclear TDP-43 staining (Fig. 6c,d).

Figure 3 | CHCHD10 depletion and FTD/ALS CHCHD10 mutations drive mitochondrial dysfunction. (a,b) Representative blots and quantification of CHCHD10 knockdown in CHCHD10 shRNA transduced HT22 cells. (c-e) Mouse hippocampus-derived HT22 neuroblastoma cells transduced with control shRNA/GFP or CHCHD10 (D10) shRNA/GFP lentivirus and stained for mitosox-red and JC-1 aggregates. (c) Representative images of mitosox-red and JC-1 aggregate staining in GFP expressing cells. Scale bar, $10 \mu \mathrm{m}$. (d,e) Quantification of average mitosox-red and JC-1 aggregate intensity per cell in GFP-positive cells ( $\geq 20$ randomly chosen cells/replicate) normalized to control ( $t$-test, $\# \# P<0.0001, n=6$ replicates). (f,g) Representative blots and quantification of CHCHD10 knockdown by CHCHD10 siRNA in HEK293T cells. (h) HEK293T cells transfected with control or CHCHD10 siRNA and subjected to qRT-PCR for the indicated mitochondria-encoded transcripts normalized to control (paired $t$-test for transcripts combined, $\# P<0.0001, n=4$ replicates). (i-I) HT22 cells co-transfected with mito-dsRed and Flag-CHCHD10 variants (WT, R15L and S59L), treated with/without FCCP (1 $\mu \mathrm{M})$ for $1 \mathrm{~h}$, stained for Flag (M2), and subjected to confocal imaging for mito-dsRed and CHCHD10 variants. (i) Representative images of mito-dsRed, and CHCHD10 (Flag M2) fluorescence. Scale bar, $5 \mu \mathrm{m}$. (j,k) Quantification of mitochondrial length without FCCP (j) and with FCCP (k) by Image J normalized to vector control (one-way ANOVA, posthhoc Tukey, ${ }^{\star} P<0.05,{ }^{\#} P<0.001,{ }^{\# \#} P<0.0001, n=6$ replicates). (I) Quantification of Flag-CHCHD10 co-localized with mito-dsRed by Image J normalized to WT control (one-way ANOVA, post hoc Tukey, ${ }^{\star} P<0.05,{ }^{\star \star} P<0.01, n=6$ replicates). ( $\mathbf{m}$ ) 293T cells transfected with control or vector control or Flag-CHCHD10 variants (WT, R15L and S59L) and subjected to qRT-PCR for the indicated mitochondria-encoded transcripts normalized to vector control

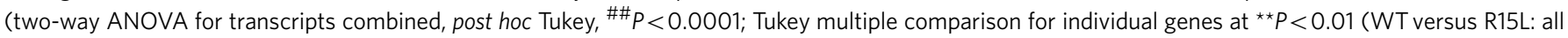
genes except COXIII; WT versus S59L: all genes; $n=6$ replicates)). All quantitative graphs with error bars were expressed as mean \pm s.e.m. ANOVA, analysis of variance. 
a

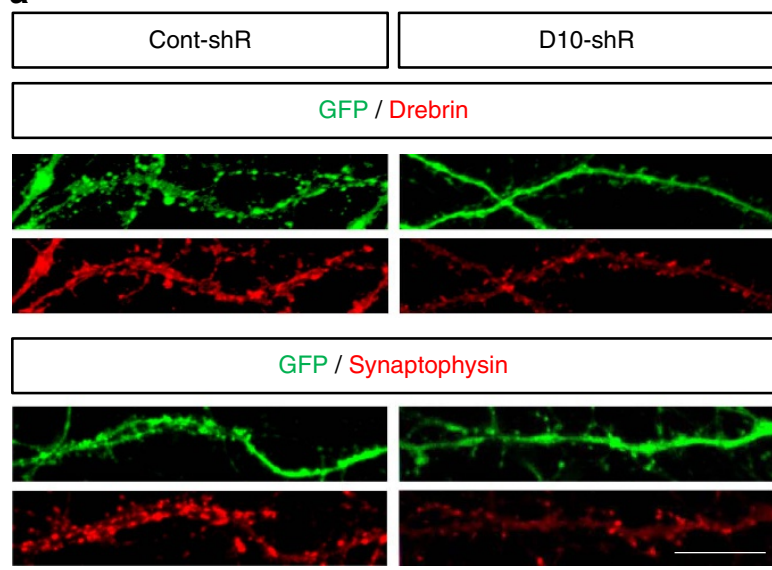

C
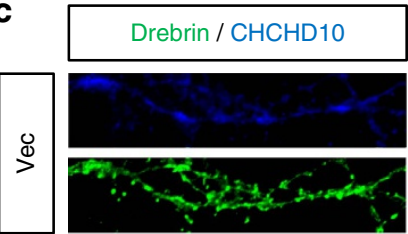

논
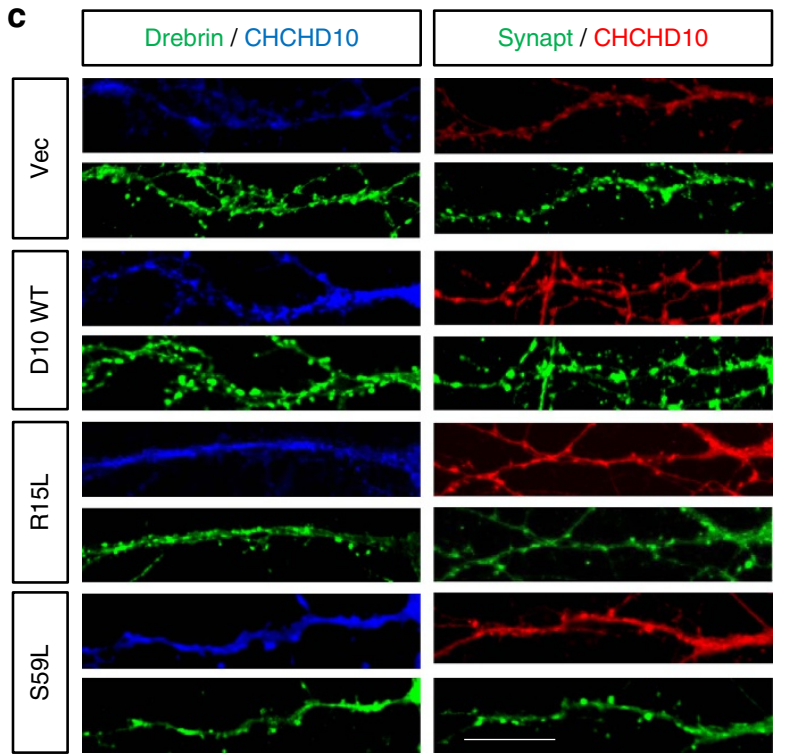

d
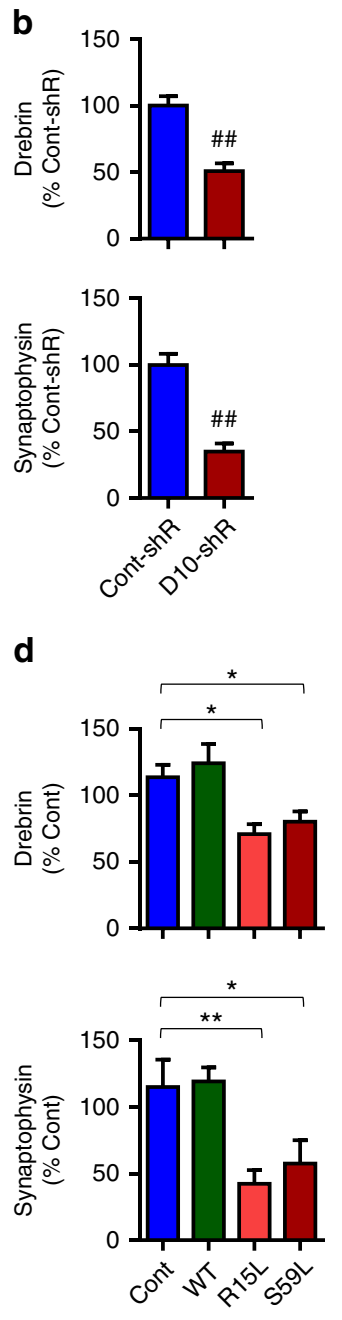

Figure 4 | CHCHD10 depletion and FTD/ALS CHCHD10 mutations compromise synaptic integrity in primary neurons. (a,c) Hippocampal primary neurons transduced with control shRNA/GFP lentivirus, CHCHD10 shRNA/GFP lentivirus or CHCHD10 rAAV9 variants (WT, R15L and S59L) and subjected to staining for drebrin, synaptophysin and/or $\mathrm{CHCHD10}$ followed by confocal imaging. Representative images of primary neurites shown. Scale bar, $20 \mu \mathrm{m}$. (b) Quantification of average overall neuritic drebrin and synaptophysin signals minus average diffuse neuritic staining (non-puncta) normalized to control in GFP ${ }^{+}$neurons ( $t$-test, ${ }^{\#} P<0.0001, n=6$ replicates, $\geq 30$ randomly chosen neurites/replicate). (d) Quantification of average overall neuritic drebrin and synaptophysin signals minus average diffuse neuritic staining (non-puncta) and normalized to control (one-way ANOVA, post hoc Tukey, ${ }^{\star} P<0.05,{ }^{\star \star} P<0.01, n=6$ replicates, $\geq 30$ randomly chosen neurites/replicate). All quantitative graphs with error bars were expressed as mean \pm s.e.m. ANOVA, analysis of variance.

To assess TDP-43 localization in a different cellular system with exogenous TDP-43 expression, NIH3T3 cells were transduced with control shRNA/GFP or CHCHD10 shRNA/GFP lentivirus and then transfected with TDP-43-tomato. Much like that seen in neurons, TDP-43-tomato was nearly exclusively nuclear in control shRNA/GFP-transduced cells (Fig. 6e). However, CHCHD10 shRNA/GFP-transduced cells exhibited significantly increased ratio of cytoplasmic/nuclear tomato-TDP-43 fluorescence (Fig. 6e,f). The cytoplasmic TDP-43-tomato exhibited punctate and aggregated appearance, which often $(\sim 45 \%)$ but not exclusively co-localized with the mitochondrial marker TOM20 (Fig. 6e,g). Biochemical solubility separation experiments showed that CHCHD10 knockdown significantly increased TDP-43-tomato in the SDS-insoluble but not in the SDS-soluble fraction (Supplementary Fig. 5a,b).

We next co-transfected NIH3T3 cells with TDP-43-tomato and Flag-CHCHD10 variants (WT, R15L and S59L) and examined TDP-43-tomato localization with mitochondria (TOM20) and
Tia-1 + granules. Cytoplasmic Tia-1 granules, so called 'stress granules', represent stress-induced punctate structures that sequester stalled mRNAs and various proteins, which is generally thought to be part of an initially protective response ${ }^{34}$. Expression of WT CHCHD10 demonstrated nearly exclusive localization of TDP-43-tomato to the nucleus (Fig. 7a). However, transfection of R15L and S59L mutations resulted in significant mislocalization of TDP-43-tomato to the cytoplasm (Fig. 7a-d). Quantification of overall TDP-43-tomato intensity in the cytoplasm versus nucleus and numbers of TDP-43-tomato puncta/cell demonstrated significant increases by R15L and S59L mutations as compared to WT control (Fig. 7b-d). These often irregularly shaped cytoplasmic TDP-43-tomato cytoplasmic puncta seen in R15L and S59L expressing cells were 35-45\% co-localized with TOM20 (Fig. 7a-d). While Tia-1 + puncta per cell were dramatically increased by R15L and S59L mutations compared to WT control (Fig. 7e,f), these mutations tended to decrease the co-localization of TDP-43-tomato with Tia-1 + granules (Fig. $7 \mathrm{~g}$ ). While either 
a

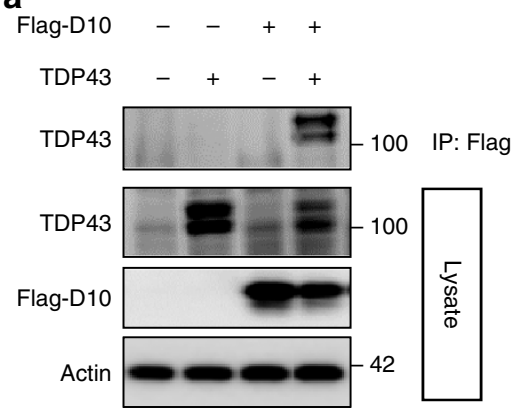

d

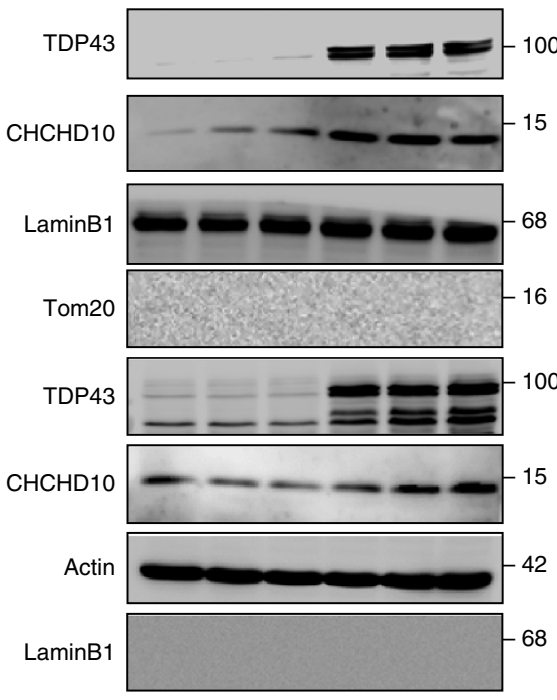

b

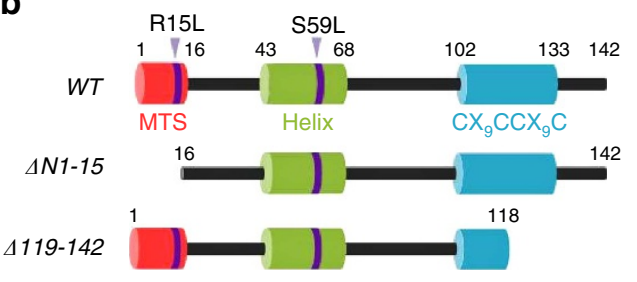

C

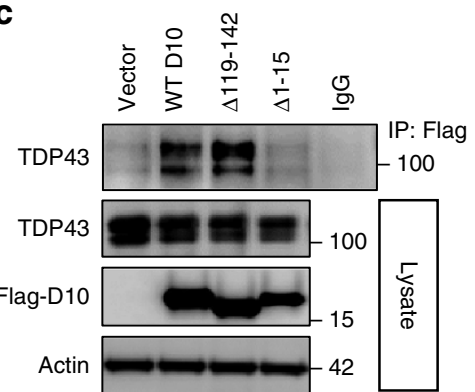

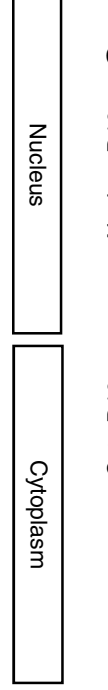
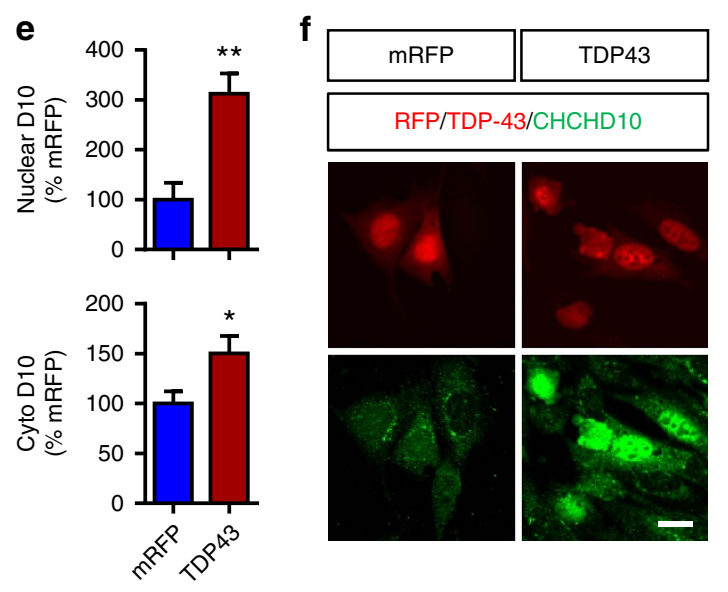

g
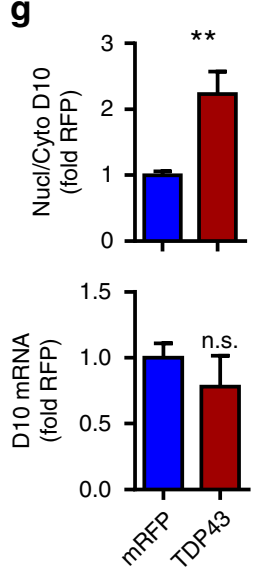
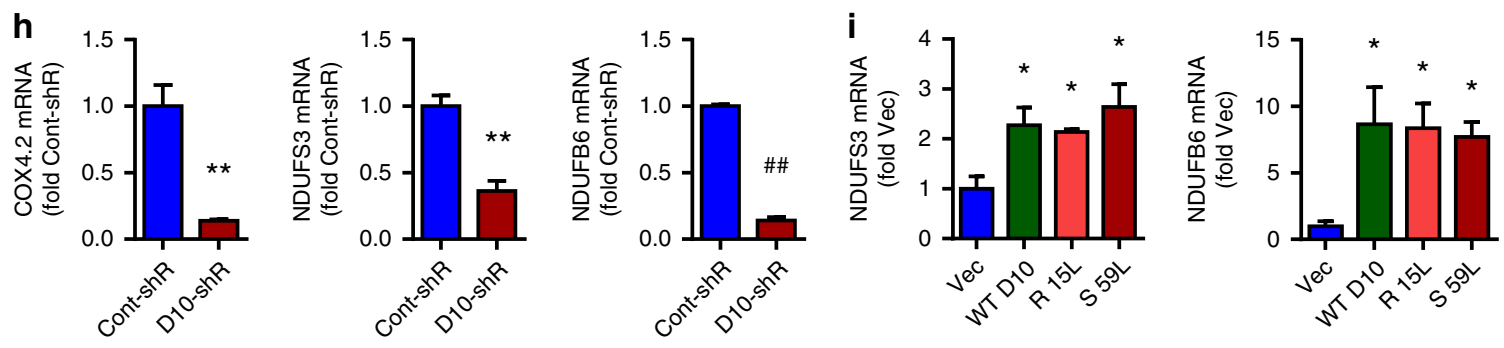

Figure 5 | TDP-43 forms a complex with CHCHD10 and promotes its nuclear localization. (a,c) HT22 cells transfected with/without TDP-43-tomato and the indicated Flag-CHCHD10 variants, and lysates subjected to co-IP with Flag (M2) antibody and/or immunoblotting for the indicated proteins. Note that the loss of N1-15 residues abrogates CHCHD10 pull down with TDP-43. (b) Schematic representation of CHCHD10 domains, mutations and deletions. (d,e) HEK293T cells transfected with mRFP or TDP-43-tomato, biochemically fractionated for nucleus versus cytoplasm, and subjected to immunoblotting for the indicated proteins. Numbers right of the blots indicate kDa markers. Note that the antibody against TDP-43 recognizes N-terminal residues 1-89, indicating that slightly faster migrating TDP-43-tomato fragment(s) likely represent cleavages(s) within the tomato fluorescent protein. (e) Quantification of nuclear and cytoplasmic TDP-43 normalized to mRFP control (t-test, ${ }^{\star} P<0.05,{ }^{\star \star} P<0.01, n=6$ replicates) (f,g) HT22 cells transfected with mRFP or TDP-43-tomato and subjected to ICC for endogenous CHCHD10 and direct fluorescence for mRFP or TDP-43-tomato. Scale bar, $10 \mu \mathrm{m}$. (g) Quantification of nuclear/cytoplasmic ratio normalized to control (top graph; $t$-test, ${ }^{\star \star} P<0.01, n=6$ replicates) and qRT-PCR for endogenous CHCHD10 transcript (bottom graph, $n=4$ replicates). (h) HT22 cells transduced with control shRNA/GFP or CHCHD10 shRNA/GFP lentivirus and subjected to qRT-PCR for

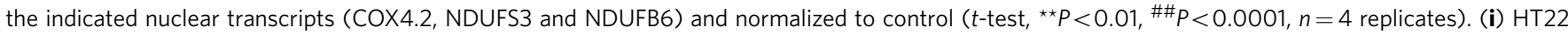
cells transfected with vector control or CHCHD10 variants (WT, R15L and S59L) and subjected to qRT-PCR for the indicated nuclear transcripts (NDUFS3 and NDUFB6) and normalized to control (one-way ANOVA, post hoc Tukey, ${ }^{\star} P<0.05$ compared to Vec, $n=4$ replicates). All quantitative graphs with error bars were expressed as mean \pm s.e.m. ANOVA, analysis of variance.

none or very few TDP-43-tomato and Tia-1 puncta were seen in WT CHCHD10 transfected cells, those cytoplasmic TDP-43tomato puncta present were largely co-localized with Tia-1+ puncta (Fig. $7 \mathrm{~g}$ ). In all cases, Tia-1 + puncta were tiny in size compared to the irregularly shaped cytoplasmic TDP-43-tomato immunoreactivity, which often co-localized with mitochondria. Intriguingly, the loss of either $\mathrm{N}$-terminal $1-15$ or C-terminal 119-142 residues of CHCHD10 S59L significantly reduced both the ratio of cytoplasmic/nuclear TDP-43-tomato and TDP-43tomato puncta per cell compared to full-length S59L, indicating 

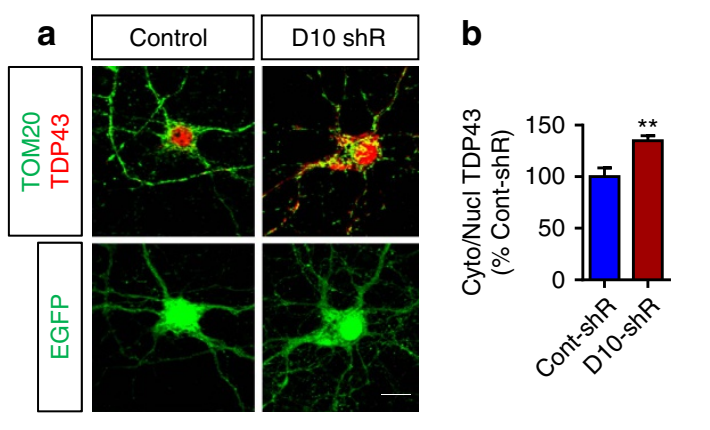

C

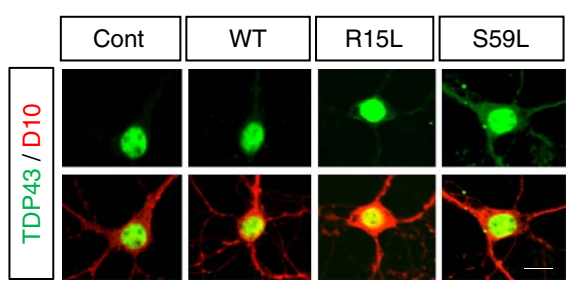

d

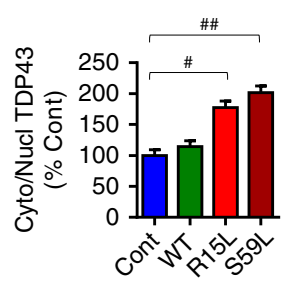

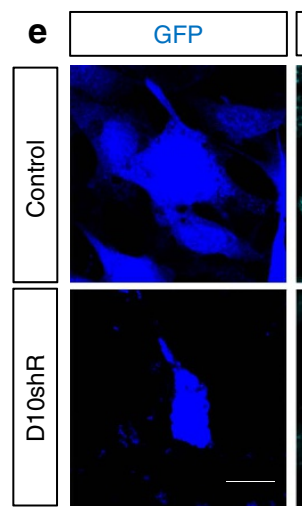
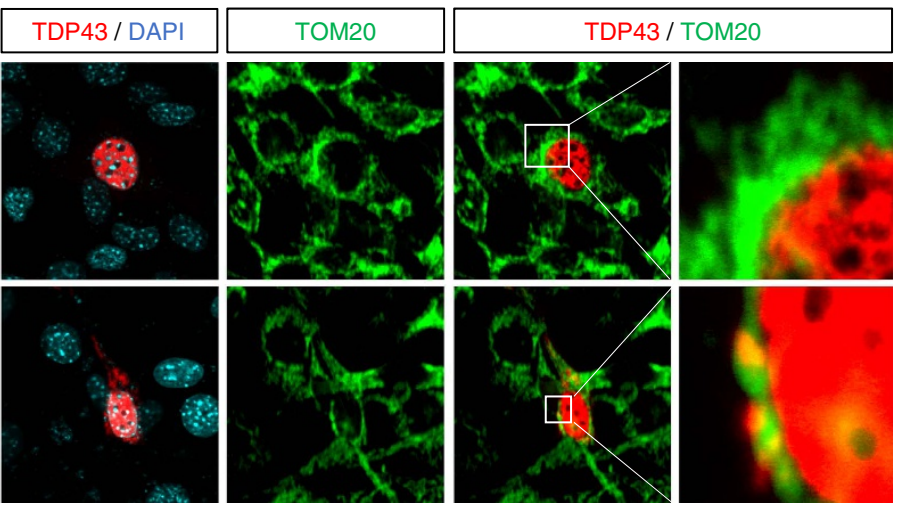

f

g
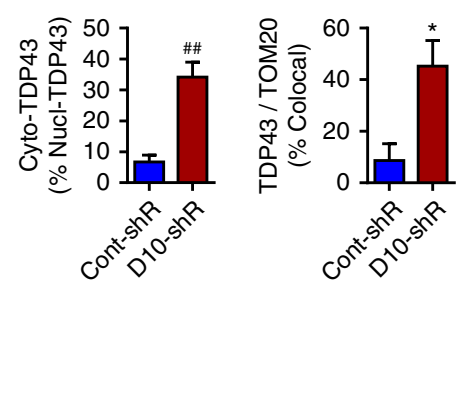

Figure 6 | CHCHD10 depletion and FTD/ALS CHCHD10 mutations induce cytoplasmic TDP-43 mislocalization. (a,b) Primary hippocampal neurons transduced with control shRNA/GFP or CHCHD10/GFP shRNA lentivirus and subjected to ICC for endogenous TDP-43 or direct GFP fluorescence (TOM20 originally blue pseudocolored to green; a, upper panels). Scale bar, $10 \mu \mathrm{m}$. (b) Quantification of cytoplasmic versus nuclear TDP-43 ratio normalized to control ( $t$-test, ${ }^{\star \star} P<0.01, n=6$ replicates). (c,d) Primary hippocampal neurons transduced with control rAAV9 or CHCHD10 rAAV9 variants and subjected to ICC for endogenous TDP-43, direct GFP fluorescence, and/or CHCHD10. Scale bar, $10 \mu \mathrm{m}$. (d) Quantification of cytoplasmic versus nuclear TDP-43 ratio normalized to control (one-way ANOVA, post hoc Tukey, ${ }^{\#} P<0.001,{ }^{\# \#} P<0.0001, n=6$ replicates). (e-g) NIH3T3 cells transduced with control shRNA/GFP or CHCHD10/GFP shRNA lentivirus and transfected with TDP-43-tomato followed by ICC for TOM20 and direct fluorescence for GFP, TDP-43-tomato, and DAPI. White outlined boxes magnified in right panels (TOM20 originally blue pseudo-coloured to green and GFP pseudocoloured to blue). Scale bar, $10 \mu \mathrm{m}$. (f,g) Quantification of cytoplasmic TDP-43 as a percentage of nuclear TDP-43 and co-localization of cytoplasmic TDP-43 with TOM20 by Image J ( $t$-test, ${ }^{\star} P<0.05,{ }^{\# \#} P<0.0001, n=6$ replicates). ANOVA, analysis of variance.

that the S59L mutation functions in concert with these domains to exhibit its pathogenic activity (Supplementary Fig. 6a,b). However, the loss of these N- and C-terminal domains did not significantly reduce cytoplasmic $\mathrm{Tia}-1+$ puncta per cell (Supplementary Fig. 6a-c). While N-terminal 16 residues of $\mathrm{CHCHD} 10$ have been hypothesized to comprise a mitochondrial targeting sequence ${ }^{28}$, deletion of either the C-terminal or $\mathrm{N}$-terminal domain significantly compromised CHCHD10 co-localization with mitochondria, although the effect of the C-terminal deletion was more pronounced (Supplementary Fig. 7a,b)

CHCHD10 variants alter TDP-43-induced apoptosis and synaptic damage. To determine the effects of $\mathrm{CHCHD} 10$ variants on TDP-43-induced cell death, we transfected HT22 cells with/ without TDP-43-tomato and Flag-CHCHD10 variants (WT, R15L or S59L) and subjected cells to Annexin V staining and alamarBlue assay, an early marker of apoptosis and a general marker of cell viability, respectively. As expected, TDP-43-tomato transfected cells exhibited a dramatic $(>2,000 \%)$ increase in Annexin $\mathrm{V}+$ cells and a significant reduction in alamarBlue OD570/600 nm ratio (Fig. 8a,b). However, co-transfection of TDP-43-tomato with WT CHCHD10 significantly ameliorated TDP-43-induced increase in Annexin V + cells and decrease in alamarBlue cell viability index, whereas both R15L and S59L mutants significantly exacerbated TDP-43-induced cell death compared to WT CHCHD10 (Fig. 8a,b). To explore this phenotype in vivo in regards to synaptic integrity, we next generated and purified high-titer $\left(>1 \times 10^{12} \mathrm{vg} \mathrm{ml}^{-1}\right)$ rAAV9 expressing mRFP control, TDP-43-tomato, or GFP-CHCHD10 variants (WT, R15L or S59L). Combinations of these purified rAAV9 were then stereotaxically injected bilaterally into the hippocampus of 2-month-old mice. One-month post injection, we processed the brains for detection of GFP-CHCHD10, TDP-43-tomato and synaptic integrity. Synaptophysin was chosen as a presynaptic marker, whereas drebrin was chosen as a postsynaptic marker. Both TDP-43-tomato and GFP-CHCHD10 variants were expressed largely within the dentate gyrus and the CA3 layer of the hippocampus (Fig. 8c). Quantification of synaptophsyin and drebrin intensity within the stratum lucidum (synaptic layer) of CA3 demonstrated that TDP-43-tomato expression dramatically reduced synaptophysin and drebrin immunoreactivity (IR) by $50 \%$ and $39 \%$, respectively (Fig. $8 \mathrm{c}-\mathrm{e}$ ). However, co-expression of TDP-43-tomato with WT CHCHD10 significantly prevented the decline in synaptophysin and drebrin IR (Fig. 8c-e). In contrast, co-expression of TDP-43-tomato with R15L or S59L mutations significantly exacerbated the decrease in synaptophysin and drebrin IR induced by TDP-43-tomato (Fig. $8 \mathrm{c}-\mathrm{e}$ ). These results taken together indicate that WT CHCHD10 blocks synaptic toxicity associated with TDP-43, whereas FTD/ALS mutants (R15L and S59L) worsen TDP-43induced synaptic pathology.

\section{Discussion}

Recent studies have shown the linkage and/or association of multiple $C H C H D 10$ mutations with the spectrum of familial and 


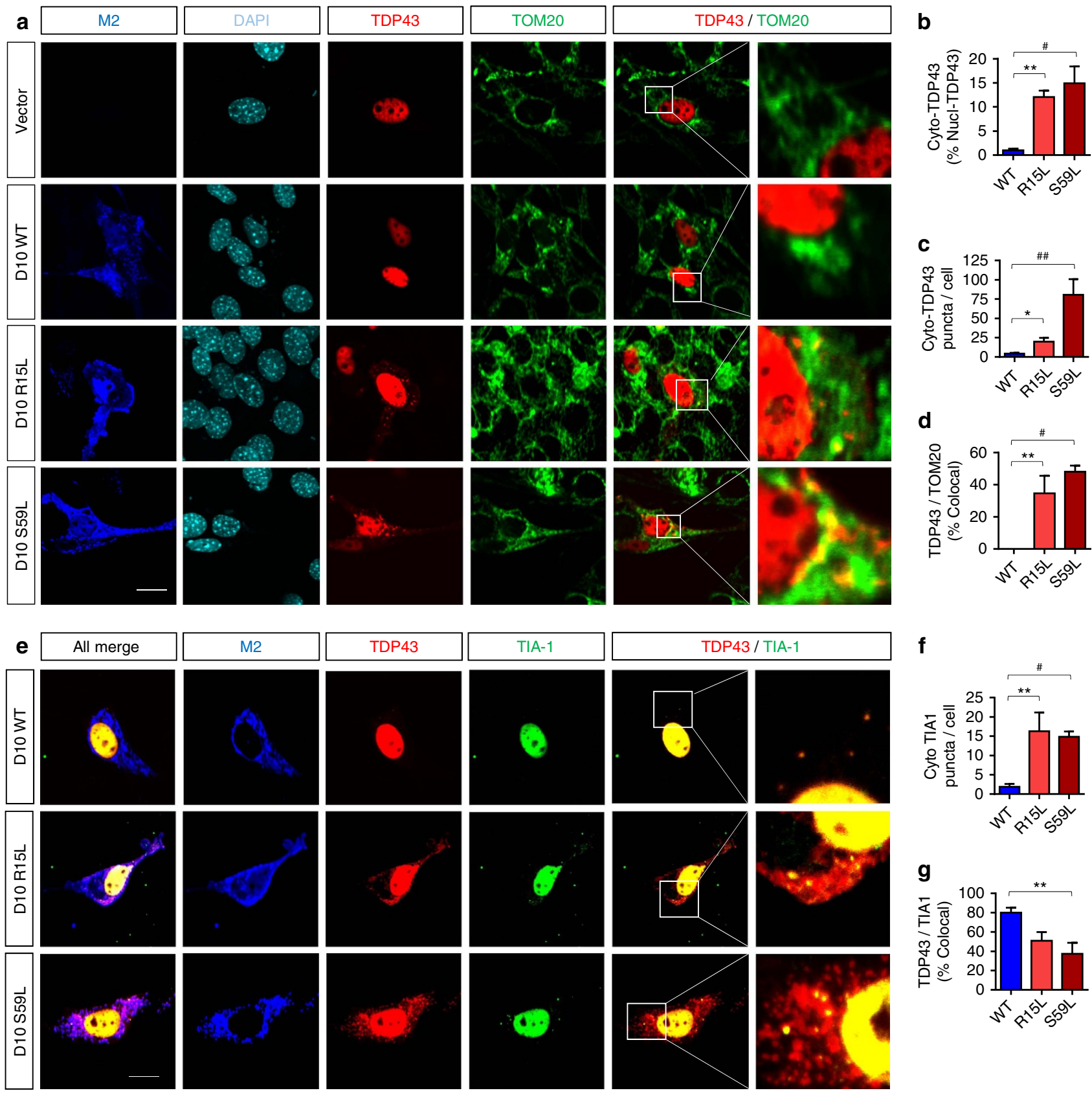

Figure 7 | FTD/ALS CHCHD10 mutations induce TDP-43 mislocalization frequently with mitochondria. (a-g) NIH3T3 cells co-transfected with

TDP43-tomato and Flag-CHCHD10 variants (WT, R15L and S59L) and subjected to ICC for Flag (M2), Tia-1, and/or TOM20 and direct fluorescence for TDP-43-tomato and DAPI. White outlined boxes magnified at far right panels. Scale bar, $10 \mu \mathrm{m}$. (b-d,f,g) Quantification of cytoplasmic TDP-43-tomato as a percentage of nuclear TDP-43-tomato, cytoplasmic TDP-43-tomato puncta per cell, co-localization of TDP-43 with TOM20 or Tia-1, and cytoplasmic Tia-1 puncta per cell (1-way ANOVA, post hoc Tukey, ${ }^{\star} P<0.05,{ }^{\star}{ }^{\star} P<0.01,{ }^{\#} P<0.001,{ }^{\#} P<0.0001, n=6$ replicates). All quantitative graphs with error bars were expressed as mean \pm s.e.m. ANOVA, analysis of varaince.

sporadic FTD-ALS diseases ${ }^{13-17}$ as well as the related CharcotMarie-Tooth disease type 2 (ref. 18) and spinal muscular atrophy Jokela type ${ }^{19}$ disorders. Despite previous observations that CHCHD10 encodes a protein largely localized to the intermembrane space of mitochondria in the MICOS complex ${ }^{14}$ and evidence that the S59L mutation reduces mitochondrial length and disorganizes mitochondrial cristae morphology ${ }^{13}$, neither the normal function of endogenous CHCHD10 nor the nature of the FTD/ALS-associated CHCHD10 mutations have been investigated. Furthermore, whether and how CHCHD10 alters the pathological milieu (that is, TDP-43 pathology) of FTD/ ALS spectrum diseases have not been scrutinized. In this study, we made a series of novel observations, utilizing C. elegans, cell lines, mouse primary neurons and mouse brains, implicating the normal protective role of $\mathrm{CHCHD} 10$ in mitochondrial and synaptic integrity, whereas FTD/ALS-associated mutations exhibit loss of function and/or dominant negative activities. Moreover, our results have for the first time tied together the activities of CHCHD10 to cytoplasmic TDP-43 inclusions, which are the principle pathological lesions in the vast majority of ALS and FTLD-TDP. Specifically, we found that C. elegans deficient in har-1, the closest orthologue to human CHCHD10, display severe abnormalities in locomotion and reduced lifespan associated with mitochondrial superoxide overproduction, 


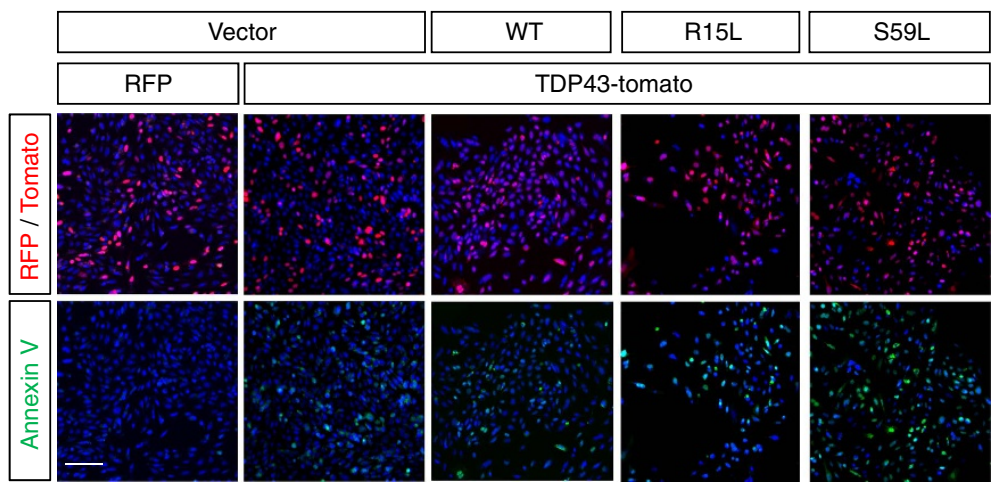

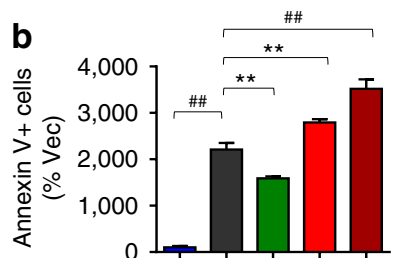

0 年,

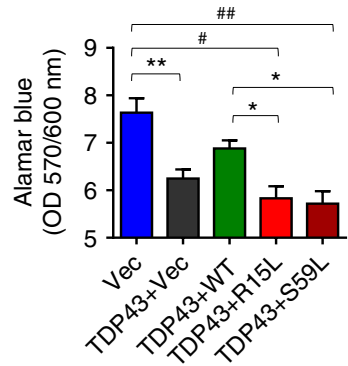

d
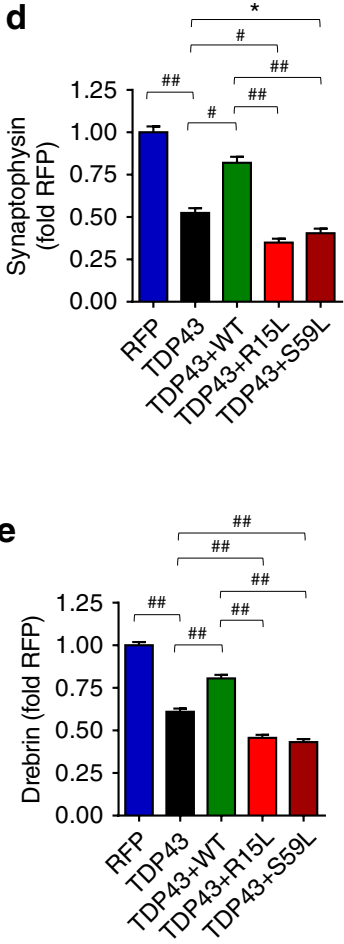

Figure 8 | Wild-type CHCHD10 ameliorates and FTD/ALS CHCHD10 mutations potentiate TDP-43-induced apoptosis and synaptic impairment. (a,b) HT22 neuroblastoma cells transfected with TDP-43-tomato and/or Flag-CHCHD10 variants, then subjected to Annexin V staining and direct TDP-43tomato fluorescence. Cells also subjected to alamarBlue staining and quantification of OD570/600 ratio. Scale bar, $100 \mu \mathrm{m}$. (b) Quantification of Annexin $\mathrm{V}+$ cells in RFP + or TDP-43-tomato + cells normalized to control (upper graph: one-way ANOVA, post hoc Tukey, ${ }^{\star \star} P<0.01,{ }^{\# \# P<0.0001, n=6}$ replicates). Quantification of alamarBlue OD570/600 ratio by microplate reader (lower graph: one-way ANOVA, post hoc Tukey, ${ }^{\star} P<0.05,{ }^{\star \star} P<0.01$, ${ }^{\#} P<0.001,{ }^{\#} P<0.0001, n=6$ replicates). (c-e) C57BL6 mice (2mo) transduced with purified high-titer TDP-43-tomato rAAV9 and/or GFP-CHCHD10 rAAV9 variants by stereotaxic injection into the hippocampus. Brains processed for direct confocal microscopy for TDP-43-tomato and GFP-CHCHD10 as well as indirect IHC for synaptophysin (presynaptic) and drebrin (postsynaptic) 1 month post injection. Scale bar, $100 \mu \mathrm{m}$. (d,e) Quantification of synaptophysin and drebrin intensity performed from the stratum lucidum (SL) of CA3 (one-way ANOVA, post hoc Tukey, ${ }^{\star} P<0.05,{ }^{\star \star} P<0.01,{ }^{\#} P<0.001$, ${ }^{\# \#} P<0.0001, n=4,5$ mice/grp). All quantitative graphs with error bars were expressed as mean \pm s.e.m. ANOVA, analysis of variance.

which were completely rescued by WT CHCHD10 but not FTD/ ALS-associated CHCHD10 mutations (R15L and S59L). In mammalian cells, overexpression of ALS/FTD-associated CHCHD10 mutations (R15L and S59L) but not WT CHDHD10 exhibited multiple abnormal mitochondrial phenotypes, nearly identical to those observed with knockdown of endogenous CHCHD10. Likewise, in primary neurons, CHCHD10 mutations (R15L and S59L) but not WT $\mathrm{CHCHD} 10$ reduced pre- and postsynaptic integrity similar to that seen by CHCHD10 knockdown. CHCHD10 formed physical complexes with TDP-43, an interaction that required intact N-terminal 16 residues, and TDP-43 promoted the nuclear localization of CHCHD10 in a retrograde signalling manner, which was associated with increased nuclear-encoded and mitochondrial targeted transcripts. Conversely, whereas WT CHCHD10 fully retained TDP-43 localization to the nucleus, FTD/ALS-associated CHCHD10 mutations (R15L and S59L) induced cytoplasmic mislocalization of TDP-43 in irregularly 
shaped inclusions often co-localized with mitochondria. Despite the significant induction of Tia-1+ granules by $\mathrm{CHCHD} 10$ mutations, large cytoplasmic TDP-43 inclusions were largely devoid of Tia-1, and the ability of CHCHD10 variants (mutations and deletions) to induce cytoplasmic TDP-43 and/or Tia-1 cytoplasmic inclusions were at least partially distinct. Finally, WT CHCHD10 antagonized TDP-43-induced apoptosis in vitro and synaptic damage in vivo, whereas FTD/ALS-associated CHCHD10 mutations (R15L and S59L) exacerbated such TDP-43-induced phenotypes. Our novel findings taken together therefore implicate the normal protective role of $\mathrm{CHCHD} 10$ in mitochondrial/synaptic integrity and inhibition of cytoplasmic TDP-43 accumulation, whereas the loss of CHCHD10 or FTD/ ALS-associated CHCHD10 mutations (R15L and S59L) exhibit loss of function or dominant negative activities in these phenotypes.

The har-1 gene in C. elegans represents the only orthologue to mammalian CHCHD10 and $C H C H D 2$, with nearly equal amino acid homology to both (http://useast.ensembl.org/index.html). Har-1 was originally identified from a C. elegans screen to detect mutants that survive in the presence of the antimitotic drug hemiasterlin, in which the har-1 G73E mutant was identified ${ }^{22}$. However, unlike the har-1 G73E mutant that exhibits a normal lifespan, har-1-1- C. elegans observed in this study were shortlived. This observation is particularly intriguing in light of the evidence that severe mitochondrial dysfunction promotes the aging process, whereas partial mitochondrial dysfunction mutants either have no effect or can activate anti-aging pathways ${ }^{22,23}$. Thus, the complete loss of har-1 appears to be more detrimental to mitochondrial health than the har-1 G73E mutation. Moreover, whereas no abnormal locomotion phenotype was noted in the har-1 G73E mutant ${ }^{22}$, the har-1 ${ }^{-1-}$ strain exhibited significant locomotion defects. Our observation that human CHCHD10 expression in the har-1 ${ }^{-1-}$ background completely rescued the locomotion and mitochondrial superoxide phenotypes indicates that the core functions of har-1 and CHCHD10 are evolutionarily conserved. However, as we used the ubiquitous eef-1A.1 promoter to drive CHCHD10 expression, it remains possible that the rescue phenotypes may not be identical to the use of the endogenous har-1 promoter. Nonetheless, the same expression level of FTD/ALS mutations (R15L and S59L) was insufficient to rescue the har-1 phenotypes, rendering them as loss of function mutations in C. elegans. This notion was supported by our observations in mammalian cells, primary neurons, and/or mouse brains that knockdown of CHCHD10 or expression of FTD/ALS CHCHD10 mutations produced nearly identical phenotypes with regards to mitochondrial and synaptic integrity. In general, the S59L mutation was more severe than the R15L mutation in most of the assays studied. Given that the S59L mutation was found in patients with both FTD and ALS symptoms ${ }^{13}$, whereas the R15L mutation was identified in patients with only ALS symptoms and some level of incomplete penetrance ${ }^{16,35}$, it is tempting to speculate that such differences may reflect the severity of phenotypes seen in this study.

The observation that multiple $\mathrm{CHCHD} 2$ mutations have recently been identified in Parkinson's ${ }^{29,30,36}$ and Lewy body disease $^{31}$ is intriguing given that CHCHD2 is a mitochondrial protein with high similarity to CHCHD10. Like CHCHD2, which can translocate from mitochondria to the nucleus to transactivate downstream mitochondrial targeted genes ${ }^{32,33}$, CHCHD10 also positively regulated COX4.2, NDUFS3 and NDUFB6 transcripts. Although this activity on NDUFS3 and NDUFB6 transcripts did not significantly differ among WT, R15L and S59L CHCHD10 variants, we cannot rule out the possibility that other CHCHD10regulated transcripts might be differentially altered by these or

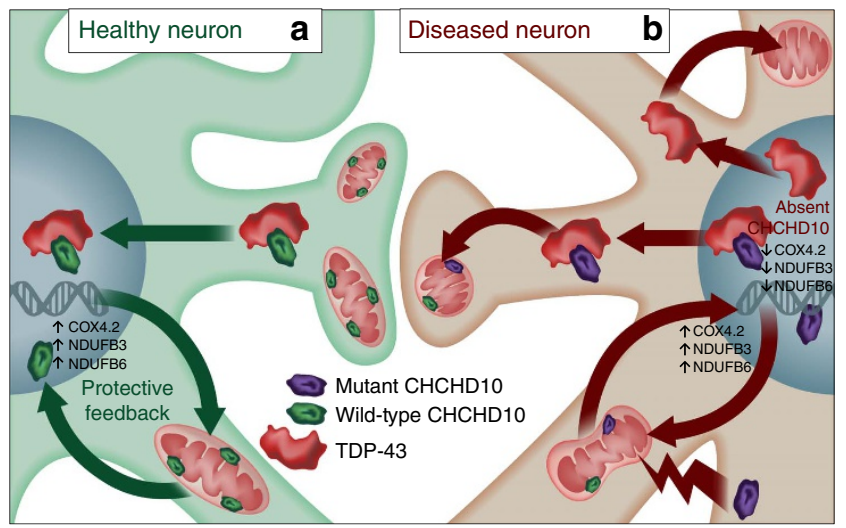

Figure 9 | Schematic model of wild-type versus absent or mutant CHCHD10 in neurons. (a) In healthy neurons (green, left), CHCHD10 is normally present in the intermembrane space of mitochondria, which functions to maintain mitochondrial morphology and synaptic integrity. Upon mitochondrial stress (that is, oxidative or hypoxic), $\mathrm{CHCHD} 10$ retrogradely signals to the nucleus, a process facilitated by its binding to TDP-43. Nuclear CHCHD10 then promotes the transcription of mitochondrial targeted nuclear genes (that is, COX4.2, NDUFB3 and NDUFB6), which facilitates rebalancing of mitochondrial function and synaptic integrity. Meanwhile, the binding of wild-type CHCHD10 to TDP-43 prevents the exit of TDP-43 from the nucleus to cytoplasm. (b) In diseased neurons (brown, right), the depletion of $\mathrm{CHCHD10}$ or expression of FTD/ALS mutant CHCHD10 fails to properly maintain mitochondrial morphology, thereby promoting mitochondrial fission and impairing synaptic integrity. The increase in mitochondrial stress also constitutively reduces mitochondrial $\mathrm{CHCHD} 10$. While mutant $\mathrm{CHCHD} 10$ is capable of binding to TDP-43, it is unable to effectively block the nuclear exit of TDP-43 from the nucleus to cytoplasm (similar to the absence of $\mathrm{CHCHD10)}$. Meanwhile, the absence of $\mathrm{CHCHD10}$ reduces the transcription of mitochondrial targeted nuclear genes, whereas the increased transcription of mitochondrial targeted nuclear genes by mutant CHCHD10 is insufficient to overcome the ongoing damage incurred in mitochondria and by cytoplasmic accumulation of TDP-43, thereby compromising synaptic integrity. In the heterozygous mutant state, FTD/ALS mutations likely function primarily in a dominant negative manner, which compromises the function of endogenous CHCHD10.

other disease-associated mutations. Nonetheless, our findings indicate that mitochondrial stress induced by the R15L or S59L mutation is likely sufficient to render neurons more vulnerable to mitochondrial and synaptic damage (Fig. 9). Although the vast majority of WT, R15L and S59L variants localized to mitochondria at steady-state, we did note a significant reduction in mitochondrial localization of $\mathrm{R} 15 \mathrm{~L}$ and $\mathrm{S} 59 \mathrm{~L}$ mutants compared to WT CHCHD10. This suggests that mitochondrial stress per se may reduce the targeting of CHCHD10 to mitochondria, after which CHCHD10 can undergo nuclear translocation to induce 'protective' nuclear transcription feedback (Fig. 9).

Accumulation of cytoplasmic TDP-43 is a key pathological feature of FTLD-TDP and ALS ${ }^{3,4}$. Previous studies have identified the stable TDP- 43 interactome to consist largely of RNA splicing factors, components of the transcriptional/ translational machinery 37,38 , as well as many mitochondrial proteins ${ }^{37}$, the latter which are involved in mitochondrial transcription/translation, electron transport and chaperone activities. Although CHCHD10 was not identified as one of these interacting proteins from mass spectrometry studies, we found that endogenous CHCHD10 does associate with endogenous TDP-43 complexes. Moreover, our findings for the first time corroborated a molecular link between FTD/ALS- 
associated CHCHD10 mutations and cytoplasmic TDP-43 accumulation. However, despite the similarity in binding of WT and mutant CHCHD10 to TDP-43, WT CHCHD10 overexpression maintained TDP-43 localization exclusively to the nucleus, suggesting that WT but not mutant CHCHD10 inhibits the exit of TDP-43 from the nucleus. This notion is supported by our finding that CHCHD10 depletion and FTD/ ALS mutations produced the same TDP-43 phenotype, indicating that the binding of CHCHD10 mutants to TDP-43 per se is not the principle cause for cytoplasmic TDP-43 accumulation. Rather, we interpret these results to indicate that FTD/ALSassociated $\mathrm{CHCHD} 10$ mutations alter the activity of the protein to act in a dominant negative manner (Fig. 9). As an example, the S59L mutation was unable to effectively promote the mislocalization of TDP-43 in the absence of either N-terminal 16 or C-terminal 119-142 residues, indicating that the S59L mutation acts in concert with these domains for its dominant negative activity. Interestingly, both domains were required for efficient mitochondrial localization of CHCHD10. We observed that $35-45 \%$ of the cytoplasmically mislocalized TDP- 43 by CHCHD10 depletion or expression of CHCHD10 mutations (R15L and S59L) was also co-localized with mitochondria. These findings are consistent with previous studies demonstrating that overexpression of TDP-43 induces mitochondrial/synaptic dysfunction, ALS-associated TDP-43 mutations localize more readily to mitochondria than its wild-type counterpart ${ }^{10,11}$, and blockade of TDP-43 mitochondrial targeting also blocks its neuronal toxicity ${ }^{12}$

It has been reported that overexpression of CHCHD10 S59L mutation in Hela cells induces mitochondrial dysfunction but inhibits apoptosis ${ }^{14}$. However in this study, we found no evidence that either R15L or S59L mutation exerted anti-apoptotic properties. Instead, WT CHCHD10 protected HT22 cells from TDP-43-induced apoptosis, whereas R15L and S59L mutations synergized with the pro-apoptotic actions of TDP-43. This potential discrepancy might be explained by the differences in the cell lines used and/or the inclusion of a specific stressor in our study, TDP-43, which was not examined in the previous study ${ }^{14}$. Nevertheless, the CHCHD10-associated apoptotic phenotype in HT22 cells commensurately mirrored the observations of synaptic integrity (synaptophysin and drebrin) in vivo, indicating that mitochondrial dysfunction exerted by CHCHD10 mutations effectively renders synapses more vulnerable in brain. Our findings taken together, therefore, provide a novel molecular linkage between the mitochondrial/synaptic toxicity of CHCHD10 dysfunction and the pathological cytoplasmic accumulation of TDP-43.

\footnotetext{
Methods

DNA constructs and siRNA. Mouse hippocampus-derived HT22 (ref. 24) and NIH3T3 (ATCC, CRL-1658) and HEK293T (ATCC, CRL-3216) cells were maintained in DMEM containing $10 \%$ FBS and $1 \%$ penicillin-streptomycin. The wtTDP43tdTomatoHA construct was a gift from Zuoshang Xu (Addgene $\# 28,205)^{7}$. The shRNA lentivirus plasmid targeting mouse CHCHD10 was purchased from Abm (\# i035693a, Richmond, BC, Canada). To generate mammalian constructs expressing human Flag-CHCHD10 variants (WT, R15L, $\Delta 1-15, \Delta 119-142)$, the CHCHD10 coding region was amplified by PCR using pyrococcus furiosus DNA polymerase (PFU) (Agilent, Santa Clara, CA, USA) and corresponding WT or mutated primers (Supplementary Table 2) from pCMVSport6-CHCHD10 (Image ID 5,747,937), subjected to restriction digest (HindIII + SalI), and inserted into p3x-Flag vector (cut with HindIII + SalI). The S59L mutant was amplified by PCR using PFU and the indicated primers (Supplementary Table 2) from pCMV-Sport6-CHCHD10, subjected to restriction digest (HindIII $+\mathrm{BmgBI}$ ), and inserted into p3x-Flag-CHCHD10 (cut with HindIII + BmgBI). To clone CHCHD10 variants into the rAAV9 plasmid, p3xFlag-CHCHD10 and its mutants were digested by SpeI and SalI, and cloned into pTR2-MCS-IG-hGFP vector at SpeI and XhoI cloning sites. TDP43-tomato-HA was cloned into pTR12.1-MCSW vector using NheI (destroyed after ligation) and XhoI restriction sites. RFP was digested by XmalI and HpaI, and cloned into
}

pTR12.1-MCSW vector using AgeI and EcoRV (destroyed after ligation) restriction sites. All cloned or subcloned plasmids were confirmed by sequencing before use in experiments. For transfections, cells were transfected for $24-48 \mathrm{~h}$ using lipofectamine 3000 according to the manufacturer's instructions (Invitrogen, Carlsbad, CA, USA). To transfect control and human CHCHD10 siRNA (5' UGAAGCAGUGCAAGUACUA-3', GE Dharmacon, Lafayette, CO, USA), 293T cells were transfected twice every $24 \mathrm{~h}$. Cells were incubated $24-48 \mathrm{~h}$ for plasmid transfections and $72 \mathrm{~h}$ for siRNA transfections before harvest.

Generation of CHCHD10 siRNA lentivirus. CHCHD10 shRNA lentivirus plasmid was purchased from Abm (\# i035693a), with the target sequence corresponding to $5^{\prime}$-CAGCCGGGTCTTATGGCTCAGATGGCATC-3'. Lentivirus vectors were co-transfected with pVSVG and pAX2 using polyethylenimine in HEK293T cells overnight. Media was removed and replaced with serum-free media for 3 days, and conditioned media was collected and centrifuged to remove cell debris. The viral medium was filtered through syringe filter $(0.45 \mu \mathrm{M})$, aliquoted and frozen in $-80^{\circ} \mathrm{C}$.

Primary neurons. Hippocampal and cortical neuron cultures were prepared from P0 mice ${ }^{24,25}$. In brief, both hippocampus and cortex were dissected separately in ice-cold HBSS and digested with trypsin. Mouse neurons were plated on glass coverslips or plates coated with poly-D-lysine (Sigma-Aldrich, St Louis, MO, USA) in neurobasal medium (Invitrogen), 2\% glutamax, and 2\% B27 supplement (Invitrogen).

Antibodies and reagents. Antibodies to CHCHD10 (1:500, 25,671-1-AP, Proteintech, Rosement, IL, USA), M2 (1:1,000, F3165, Sigma-Aldrich), TOM20 (1:1,000, FL-145, Santa Cruz Biotechnology, Dallas, TX, USA), Drebrin (1:500, ab12,350, Abcam, Cambridge, MA, USA), Synaptophysin (1:500, S5768, Sigma-Aldrich), TDP-43 (1:500, MABN45, Merck Millipore, Darmstadt, Germany), LaminB1 (1:1,000, D4Q4Z, Cell Signaling, Danvers, MA, USA), Actin (1:3,000, A5316, Sigma-Aldrich), Actin (1:1,000, sc-1,616, Santa Cruz, Dallas TX, USA), tRFP (1:1,000, AB233, Evrogen, Moscow, Russia), horseradish peroxidase-linked secondary antibodies (1:5,000, Jackson ImmunoResearch, West Grove, PA, USA), and fluorescently labelled secondary antibodies (1:1,000, Invitrogen) were obtained from the indicated sources.

Cell lysis and immunoblotting. Cells were lysed using RIPA lysis buffer ( $50 \mathrm{mM}$ Tris pH 7.4, $150 \mathrm{mM} \mathrm{NaCl}, 2 \mathrm{mM}$ ethlenediaminetetraacetic acid, $1 \%$ NP-40, $0.1 \%$ sodium dodecyl sulfate). For co-immunoprecipitation experiments, cells were lysed in NP-40 lysis buffer $(50 \mathrm{mM}$ Tris $\mathrm{pH} 7.4,150 \mathrm{mM} \mathrm{NaCl}, 2 \mathrm{mM}$ ethlenediaminetetraacetic acid, 1\% NP-40). Biochemical nuclear versus cytoplasmic isolation was performed using the nuclear isolation kit for cells (Thermo Scientific, Rockford, IL, USA) according to the manufacturer's instructions. For SDS-insoluble fractions, pellets from RIPA-insoluble lysates were extracted with $4 \times$ lithium dodecyl sulfate (LDS) sample buffer followed by sonication and boiling. Total protein concentrations were quantified by a colorimetric detection assay (BCA Protein Assay, Pierce, Rockford, IL, USA). Equal amounts of protein lysates were separated by SDS-polyacrylamide gel electrophoresis, and transferred to Immobilon-P membranes (Merck Millipore, Darmstadt, Germany). Proteins of interest were probed by primary antibodies and corresponding peroxidase-conjugated secondary antibodies, followed by detection by ECL (Merck Millipore, Darmstadt, Germany) and capture using the LAS-4000 imager (GE Healthcare Biosciences, Pittsburgh, PA, USA). Images have been cropped for presentation. Full-length blot images with molecular weight markers are presented in Supplementary Figs 8-12.

Quantitative real-time RT-PCR. Quantitative real-time RT-PCR was performed using Roche LightCycler 96 System (Life Science, San Francisco, CA, USA), following the manufacturer's recommended conditions. Total RNA was isolated from transiently transfected cells using Trizol reagent (Invitrogen), reverse transcribed (Superscript III, Invitrogen), and subjected to quantitative PCR analysis using Syber green master mix (Invitrogen). The comparative threshold cycle $(\mathrm{Ct})$ value was used to calculate the amplification factor, and the relative amount of targets was normalized to GAPDH levels ${ }^{39}$. The primer sequences for each target transcript are provided in Supplementary Tables 3 and 4.

Mitochondrial health and apoptosis assays. HT22 cells were plated onto $35-\mathrm{mm}$ glass bottom dishes or 24-well plates with glass coverslips (both coated with fibronectin). After $24 \mathrm{~h}$, cells were either transduced with lentivirus or transfected with indicated plasmids for up to $48 \mathrm{~h}$. For Annexin V (BD Biosciences, San Jose, CA, USA), JC-1 (Invitrogen), and MitoSox-Red (Invitrogen) staining, live cells were washed with PBS and stained with Annexin V-FITC, JC-1, MitoSox-Red for $20 \mathrm{~min}$. Cells were washed one time with binding buffer, and images were captured by Olympus FV10i confocal microscope (Tokyo, Japan) ${ }^{24}$. AlamarBlue cell viability assays (Invitrogen) were performed per the manufacturer's instructions in a microtiter plate reader with absorbance reading at $570 \mathrm{~nm}$, using $600 \mathrm{~nm}$ as a reference wavelength for normalization. 
Immunocytochemistry and Immunohistochemistry. For immunocytochemistry (ICC), cells were fixed using $4 \%$ paraformaldehyde for $15 \mathrm{~min}$ at room temperature. Cells were blocked using 3\% BSA with $0.1 \%$ Triton-x-100 for $1 \mathrm{~h}$ and subjected in primary antibodies incubation for overnight at $4{ }^{\circ} \mathrm{C}$ and secondary antibody (Alexa-405, Alexa-488, or Alexa-594, Invitrogen) incubation for $1 \mathrm{~h}$ at room temperature, followed by counterstaining with DAPI and mounting. For immunohistochemistry (IHC), mice were perfused with PBS, and half brains were immediately stored at $-80^{\circ} \mathrm{C}$ for biochemical analysis, and the other half was fixed with $4 \%$ paraformaldehyde at $4{ }^{\circ} \mathrm{C}$ for $24 \mathrm{~h}$ followed by cryoprotection in $30 \%$ sucrose. Thirty-micron sections were blocked using normal goat serum for $1 \mathrm{~h}$ and subjected to primary antibodies (anti-drebrin 1:100 Abcam, Cambridge, MA, USA; or anti-synaptophysin $1: 100$, Sigma) at $4{ }^{\circ} \mathrm{C}$ for overnight, followed by secondary antibody (Alexa-405 and Alexa-488) incubation for $1 \mathrm{~h}$ at room temperature before mounting. Images were captured with the Olympus FV10i confocal microscope, and the immunoreactivities were quantified from hippocampus CA3 region using the Image J software (National Institutes of Health, Bethesda, MD, USA). Immunoreactivities were quantitated from every 12th serial section through an entire hippocampus. In ICC and IHC experiments, all comparison images were acquired with identical laser intensity, exposure time, and filter. Adjustments to the brightness/contrast were applied equally to all comparison images. Regions of interest were chosen randomly, and investigators were blinded to experimental conditions during image acquisition and quantification.

Generation of rAAV9 and stereotaxic injections in mice. Recombinant AAV9 viruses were generated by co-transfection of serotype vector expressing the interest gene with pAAV9 and pXX6 in HEK293 cells and subjected to purification as described ${ }^{40}$. C57BL6 mice were supplied with water and food ad libitum with 12-hour light/dark cycle at standard vivarium conditions. For brain injections, isoflurane anaesthetised mice (2-month old, equally balanced for gender per condition) were placed onto the stereotaxic apparatus, and burr holes were drilled using a dental drill bit (SSW HP-3; SSWhite Burs, Lakewood, NJ, USA) through the cranium. Bilateral injections were made into the hippocampus with a 26 gauge needle attached to a 10- $\mu$ l syringe (Hamilton, Reno, NV, USA) at the following coordinates: anteroposterior $-2.7 \mathrm{~mm}$, lateral $-2.7 \mathrm{~mm}$, and vertical $3.0 \mathrm{~mm}$. A total volume of $2 \mu \mathrm{l}$ purified rAAV $\left(1 \times 10^{12} \mathrm{vg} \mathrm{ml}^{-1}\right)$ was injected over a $2 \mathrm{~min}$ period using the convection enhanced delivery method ${ }^{40}$. The incision was then cleaned and closed with surgical sutures. The mice recovered within $20 \mathrm{~min}$ and were housed singly until the time they were sacrificed 4 weeks post injection.

C. elegans transgenesis. To generate the BFP fusion $\mathrm{CHCHD} 10$ variants, $\mathrm{Mul}-$ tisite Gateway cloning entry vectors containing promoter eef-1A.1 (peef-1A.1), fulllength CHCHD10 variants (wild-type, R15L and S59L) with stop codon removed, and tagBFP::unc-54 3'-untranslated region (UTR) were amplified via Phusion High-Fidelity DNA Polymerase (NEB) and cloned into pDONRP4-P1r, pDONR221 and pDONRP2R-P3, respectively. To generate attB4/attB1r flanked peef-1A.1, Gateway-compatible primers were used to amplify peef-1A.1 from pCFJ601 (Addgene). att flanked CHCHD10 variants were subcloned from p3xFlag-CHCHD10 variants (WT, R15L and S59L) before BP cloning. Lastly, to specifically generate 'tagBFP::unc-54 3'-UTR', 24 base pair overlapping PCR products of tagBFP amplified from pSR04 (Addgene) and unc-54 $3^{\prime}$-UTR amplified from pCFJ104 (Addgene) were generated and PCR stitching was used to fuse the att flanked product before BP cloning ${ }^{41}$. Three expression clones were generated containing peef-1A.1, CHCHD10 (wild-type, R15L or S59L), and tagBFP::unc-54 $3^{\prime}$-UTR by LR reaction with pCG150 (Addgene). The three resulting expression constructs were fully sequence-verified before microinjection.

C. elegans strains and microinjections. Unless otherwise stated, the C. elegans strains used were obtained from the Caenorhabditis Genetics Center (University of Minnesota): wild-type Bristol N2, har-1(gk3124) III, and $d v I s 62 \mathrm{X}$. The har$1(\mathrm{gk} 3124)$ strain was provided by the C. elegans Gene Knockout Project at the Oklahoma Medical Research Foundation, which was part of the International C. elegans Gene Knockout Consortium. Transgenic strains used in this study including CHCHD10 variants (WT, R15L and S59L) and their corresponding CGC designations are listed in Supplementary table 1 . Strains were maintained at $16^{\circ} \mathrm{C}$ on nematode growth media (NGM) agar plates with OP50 Escherichia coli unless otherwise indicated. Experimental plates were age-synchronized using timed egg laying ${ }^{42}$. Transgenic C. elegans strains expressing wild-type or mutant hCHCHD10 variants were generated by microinjection of har-1 knockout animals, har1 (gk3124) III, with peef-1A.1::CHCHD10 (wild-type, R15L or S59L)::BFP::unc-54 $3^{\prime}$-UTR $\left(50 \mathrm{ng} \mu \mathrm{l}^{-1}\right)$, and BFP positive animals were selected for at least three generations before experimentation. All microinjections were performed by Knudra Transgenics (Murray, UT, USA). Multiple lines per transgene were generated and propagated separately. All strains are available upon request.

C. elegans locomotion assays. Motility. Age-synchronized worms per strain (3 day adults) were transferred to a fresh NGM plate, exposed to room temperature for $30 \mathrm{~min}$, and then videotaped for $1 \mathrm{~min}$ at room temperature. Worm motility (body lengths per second, BLPS) was measured using the ImageJ Plugin wrMTrck (Jesper Sondergaard Pedersen) ${ }^{43}$.
Thrashing. Age-synchronized worms per strain (3 day adults) were transferred to room temperature $\mathrm{M} 9$ buffer and recorded $2 \mathrm{~min}$ after introduction to liquid for $1 \mathrm{~min}$. Thrashing speed (BBPS) was measured manually with a click counter. Per cent time spent curling was measured manually as the number of frames spent curled per total number of frames by a blinded investigator.

C. elegans mitosox-red imaging. Age-synchronized worms per strain (3 day adults) were transferred to fresh NGM plates without $E$. coli and allowed to crawl on plates for $15 \mathrm{~min}$ at $16^{\circ} \mathrm{C}$ to clear their gut. Afterwards, worms were incubated in $\mathrm{M} 9$ Buffer with $10 \mathrm{mM}$ sodium azide and $5 \mu \mathrm{M}$ mitosox-red (Invitrogen) for $30 \mathrm{~min}$ at room temperature, mounted live on agar pads, and imaged immediately with an Olympus FV10i confocal microscope. Average intensity of head region excluding mouth, pharynx and intestine was quantified with ImageJ (NIH ImageJ, Bethesda, MD, USA) in a blinded fashion.

C. elegans lifespan assay. L4 stage age-synchronized worms per strain were transferred to OP50 plates and kept at $16^{\circ} \mathrm{C}$. Number of dead worms per plate was scored each day by a blinded observer. Worms were assumed dead if they remained motionless after prodding with an eyebrow pick. Living worms were transferred to fresh plates every 2 days until there were no surviving worms.

C. elegans immunoblotting. Well-fed worms were washed three times with M9 buffer and then lysed using repeated freeze-thaw and sonication in a lysis buffer (50 mM Tris-Cl, $150 \mathrm{mM} \mathrm{NaCl}, 2 \mathrm{mM}$ EDTA, and 1\% Triton X-100 supplemented with protease and phosphatase inhibitor cocktails) and centrifuged at $130,000 \mathrm{~g}$ for $5 \mathrm{~min}$. Protein quantification of the soluble sample was performed by a colorimetric detection reagent (BCA protein assay, Pierce). Equal amounts of protein were subjected to SDS-polyacrylamide gel electrophoresis and transferred to nitrocellulose membranes for immunoblotting. After probing with primary antibody (anti-actin (I-19), Santa Cruz, Dallas, TX, USA, anti-CHCHD10 Proteintech; antitRFP Evrogen), the corresponding peroxidase-conjugated secondary antibody was detected by ECL western blot reagents (Pierce). ECL images were captured by the Fuji/GE LAS-4,000 imager (LAS-4,000, Pittsburgh, PA, USA) and quantified using ImageJ software (NIH ImageJ).

Statistical analysis and graphs. Statistical data were analysed by GraphPad Prism 6.0 software (GraphPad Software, San Diego, CA, USA) using Student's $t$-test, oneor two-way analysis of variance. One- or two-way analysis of variance was followed by the indicated post hoc tests. Survival analysis was performed by the Log-rank (Mantel-Cox) test. All quantitative graphs with error bars were expressed as mean \pm s.e.m.

Data availability. The data that support the findings of this study are available from the corresponding authors on reasonable request.

\section{References}

1. Burrell, J. R. et al. The frontotemporal dementia-motor neuron disease continuum. Lancet 388, 919-931 (2016).

2. Ng, A. S., Rademakers, R. \& Miller, B. L. Frontotemporal dementia: a bridge between dementia and neuromuscular disease. Ann. N. Y. Acad. Sci. 1338, 71-93 (2015).

3. Weishaupt, J. H., Hyman, T. \& Dikic, I. Common molecular pathways in amyotrophic lateral sclerosis and frontotemporal dementia. Trends Mol. Med. 22, 769-783 (2016).

4. Janssens, J. \& Van Broeckhoven, C. Pathological mechanisms underlying TDP-43 driven neurodegeneration in FTLD-ALS spectrum disorders. Hum. Mol. Genet. 22, R77-R87 (2013).

5. Buratti, E. Functional significance of TDP-43 mutations in disease. Adv. Genet 91, 1-53 (2015).

6. Igaz, L. M. et al. Expression of TDP-43 C-terminal Fragments in vitro recapitulates pathological features of TDP-43 proteinopathies. J. Biol. Chem. 284, 8516-8524 (2009).

7. Yang, C. et al. The C-terminal TDP-43 fragments have a high aggregation propensity and harm neurons by a dominant-negative mechanism. PLoS ONE 5, e15878 (2010)

8. Zhang, Y. J. et al. Aberrant cleavage of TDP-43 enhances aggregation and cellular toxicity. Proc. Natl Acad. Sci. USA 106, 7607-7612 (2009).

9. Josephs, K. A. et al. TAR DNA-binding protein 43 and pathological subtype of Alzheimer's disease impact clinical features. Ann. Neurol. 78, 697-709 (2015).

10. Wang, W. et al. The ALS disease-associated mutant TDP-43 impairs mitochondrial dynamics and function in motor neurons. Hum. Mol. Genet. 22, 4706-4719 (2013).

11. Xu, Y. F. et al. Wild-type human TDP-43 expression causes TDP-43 phosphorylation, mitochondrial aggregation, motor deficits, and early mortality in transgenic mice. J. Neurosci. 30, 10851-10859 (2010).

12. Wang, W. et al. The inhibition of TDP-43 mitochondrial localization blocks its neuronal toxicity. Nat. Med. 22, 869-878 (2016). 
13. Bannwarth, S. et al. A mitochondrial origin for frontotemporal dementia and amyotrophic lateral sclerosis through CHCHD10 involvement. Brain 137, 2329-2345 (2014).

14. Genin, E. C. et al. CHCHD10 mutations promote loss of mitochondrial cristae junctions with impaired mitochondrial genome maintenance and inhibition of apoptosis. EMBO Mol. Med. 8, 58-72 (2016).

15. Johnson, J. O. et al. Mutations in the CHCHD10 gene are a common cause of familial amyotrophic lateral sclerosis. Brain 137, e311 (2014).

16. Muller, K. et al. Two novel mutations in conserved codons indicate that CHCHD10 is a gene associated with motor neuron disease. Brain 137, e309 (2014).

17. Zhang, M. et al. Mutation analysis of CHCHD10 in different neurodegenerative diseases. Brain 138, e380 (2015).

18. Penttila, S. et al. Late onset spinal motor neuronopathy is caused by mutation in CHCHD10. Ann. Neurol. 77, 163-172 (2015).

19. Auranen, M. et al. CHCHD10 variant p.(Gly66Val) causes axonal CharcotMarie-Tooth disease. Neurol. Genet. 1, el (2015).

20. Ash, P. E. et al. Neurotoxic effects of TDP-43 overexpression in C. elegans. Hum. Mol. Genet. 19, 3206-3218 (2010).

21. Restif, C. et al. CeleST: computer vision software for quantitative analysis of C. elegans swim behaviour reveals novel features of locomotion. PLoS Comput. Biol. 10, e1003702 (2014)

22. Zubovych, I. O., Straud, S. \& Roth, M. G. Mitochondrial dysfunction confers resistance to multiple drugs in Caenorhabditis elegans. Mol. Biol. Cell 21 956-969 (2010)

23. Wang, Y. \& Hekimi, S. Mitochondrial dysfunction and longevity in animals: untangling the knot. Science 350, 1204-1207 (2015).

24. Woo, J. A. et al. Slingshot-Cofilin activation mediates mitochondrial and synaptic dysfunction via Abeta ligation to beta1-integrin conformers. Cell Death Differ. 22, 921-934 (2015)

25. Woo, J. A. et al. RanBP9 at the intersection between cofilin and Abeta pathologies: rescue of neurodegenerative changes by RanBP9 reduction. Cell Death Dis. 6, 1676 (2015).

26. Smiley, S. T. et al. Intracellular heterogeneity in mitochondrial membrane potentials revealed by a J-aggregate-forming lipophilic cation JC-1. Proc. Natl Acad. Sci. USA 88, 3671-3675 (1991).

27. Sheng, Z. H. \& Cai, Q. Mitochondrial transport in neurons: impact on synaptic homeostasis and neurodegeneration. Nat. Rev. Neurosci. 13, 77-93 (2012).

28. Cozzolino, M., Rossi, S., Mirra, A. \& Carri, M. T. Mitochondrial dynamism and the pathogenesis of amyotrophic lateral sclerosis. Front. Cell Neurosci. 9, 31 (2015).

29. Funayama, M. et al. CHCHD2 mutations in autosomal dominant late-onset Parkinson's disease: a genome-wide linkage and sequencing study. Lancet Neurol. 14, 274-282 (2015).

30. Ogaki, K. et al. Mitochondrial targeting sequence variants of the $\mathrm{CHCHD} 2$ gene are a risk for Lewy body disorders. Neurology 85, 2016-2025 (2015).

31. Shi, C. H. et al. CHCHD2 gene mutations in familial and sporadic Parkinson's disease. Neurobiol. Aging 38, 217 e219-213 (2016).

32. Aras, S. et al. Oxygen-dependent expression of cytochrome c oxidase subunit 4-2 gene expression is mediated by transcription factors RBPJ, CXXC5 and CHCHD2. Nucleic Acids Res. 41, 2255-2266 (2013).

33. Aras, S. et al. MNRR1 (formerly CHCHD2) is a bi-organellar regulator of mitochondrial metabolism. Mitochondrion 20, 43-51 (2015).

34. Waris, S., Wilce, M. C. \& Wilce, J. A. RNA recognition and stress granule formation by TIA proteins. Int. J. Mol. Sci. 15, 23377-23388 (2014).

35. Kurzwelly, D., Kruger, S., Biskup, S. \& Heneka, M. T. A distinct clinical phenotype in a German kindred with motor neuron disease carrying a CHCHD10 mutation. Brain 138, e376 (2015)

36. Funayama, M. \& Hattori, N. CHCHD2 and Parkinson's disease--authors' reply. Lancet Neurol. 14, 682-683 (2015).

37. Freibaum, B. D., Chitta, R. K., High, A. A. \& Taylor, J. P. Global analysis of TDP-43 interacting proteins reveals strong association with RNA splicing and translation machinery. J. Proteome Res. 9, 1104-1120 (2010).
38. Ling, S. C. et al. ALS-associated mutations in TDP-43 increase its stability and promote TDP-43 complexes with FUS/TLS. Proc. Natl Acad. Sci. USA 107, 13318-13323 (2010).

39. Liu, K., Zhuang, X. \& Mai, Z. p73 expression is associated with cellular chemosensitivity in human non-small cell lung cancer cell lines. Oncol. Lett. 5, 583-587 (2013).

40. Carty, N. et al. Convection-enhanced delivery and systemic mannitol increase gene product distribution of AAV vectors 5, 8, and 9 and increase gene product in the adult mouse brain. J. Neurosci. Methods 194, 144-153 (2010).

41. Shevchuk, N. A. et al. Construction of long DNA molecules using long PCRbased fusion of several fragments simultaneously. Nucleic Acids Res. 32, e19 (2004).

42. Sutphin, G. L. \& Kaeberlein, M. Measuring Caenorhabditis elegans life span on solid media. J. Vis. Exp. 27, 1152 (2009).

43. Nussbaum-Krammer, C. I., Neto, M. F., Brielmann, R. M., Pedersen, J. S. \& Morimoto, R. I. Investigating the spreading and toxicity of prion-like proteins using the metazoan model organism C. elegans. J. Vis. Exp. 95, 52321 (2015).

\section{Acknowledgements}

Some strains were provided by the CGC, which is funded by NIH Office of Research Infrastructure Programs (P40 OD010440). This work was supported in part by grants from the VA (1 I01 BX002478-01A1, D.E.K.), NIH (1 R21 AG050284-01, D.E.K.) and Florida department of Health (5AZ07, D.E.K.).

\section{Author contributions}

J.-A.A.W. contributed to Figs 3-7. T.L. contributed to Figs 3,5 and 8. C.T. contributed to Figs 1,2 and 9. C.C.F. contributed to Figs 6 and 7. E.D.N. and A.B. contributed to Fig. 8 P.L. contributed to Figs 3,5 and 8. X.Z. generated all Flag-CHCHD10 plasmids. A.D. and S.D.W. contributed to Fig. 2. D.E.K. contributed to all figures. All authors contributed to data analysis and review of the manuscript.

\section{Additional information}

Supplementary Information accompanies this paper at http://www.nature.com/ naturecommunications

Competing interests: The authors declare no competing financial interests.

Reprints and permission information is available online at http://npg.nature.com/ reprintsandpermissions/

How to cite this article: Woo, J.-A. A. et al. Loss of function CHCHD10 mutations in cytoplasmic TDP-43 accumulation and synaptic integrity. Nat. Commun. 8, 15558 doi: $10.1038 /$ ncomms15558 (2017).

Publisher's note: Springer Nature remains neutral with regard to jurisdictional claims in published maps and institutional affiliations.

This work is licensed under a Creative Commons Attribution 4.0 International License. The images or other third party material in this article are included in the article's Creative Commons license, unless indicated otherwise in the credit line; if the material is not included under the Creative Commons license, users will need to obtain permission from the license holder to reproduce the material. To view a copy of this license, visit http://creativecommons.org/licenses/by/4.0/

C) The Author(s) 2017 